\title{
DS-CDMA Receiver Based on a Five-Port Technology
}

\author{
Ivo Maljević \\ The Edward S. Rogers Sr. Department of Electrical and Computer Engineering, University of Toronto, Toronto, ON, Canada M5S 1A1 \\ Email: ivom@comm.utoronto.ca
}

\author{
Elvino S. Sousa \\ The Edward S. Rogers Sr. Department of Electrical and Computer Engineering, University of Toronto, Toronto, ON, Canada M5S 1A1 \\ Email:sousa@comm.utoronto.ca
}

Received 29 February 2004; Revised 15 November 2004

\begin{abstract}
High data rates, low-power consumption, and low complexity will be the most important parameters in the design of the nextgeneration mobile terminals. In this paper we are introducing a new paradigm in the design of direct sequence spread spectrum receiver by combining analog and digital signal processing. The main difference with respect to the conventional all-digital receiver design approach is that the proposed mixed analog/digital processing results in a symbol rate sampling rather than the high-rate subchip sampling. Analog signal despreading is the key part of the proposed receiver solution, which is based on a five-port device, a passive RF square-law-type device. It is used to perform two important tasks at the same time, namely, the direct conversion and analog despreading. To achieve lower complexity, the proposed receiver uses rectangular instead of pulse-matched despreading at the cost of only a small performance degradation. Also, we propose a new noncoherent pseudonoise (PN) code tracking scheme based on error signal generated through the L1 norm. This results in comparable or even better PN code tracking performance than L2 norm circuitry, using less complex hardware. Further, we explore how this technology can be applied in the design of DS-CDMA RAKE receiver for mobile terminals. Depending on how the pilot signal is multiplexed, we propose two types of RAKE receivers. It is shown that under Rayleigh fading channel such receiver structures offer robustness and high performance, while maintaining the low complexity achievable through the five-port device.
\end{abstract}

Keywords and phrases: five-port device, CDMA receiver, direct conversion, RAKE receiver.

\section{INTRODUCTION}

When it comes to designing wireless receivers, issues of complexity and low-power consumption have become as important as achieving high performance. Besides the all-digital approach that addresses the performance issue, the old, yet improved, analog design approach is beginning to emerge. Hagenauer et al. [1] have demonstrated that it is possible to implement data detection, equalization, and decoding in analog VLSI circuits, resulting in a significant speed gain and lower power consumption. In an in-depth overview [2] of a low-power WCDMA system design, that also relies on analog processing, it has been shown that direct sequence is well suited for a multiple access system in terms of power consumption. It has been estimated [3] that more than 50\% of the total processing power in DS-CDMA receivers is spent on despreading. Not surprisingly, a majority of work on lowpower consumption is focused on a correlator design. For instance, analog implementation of a baseband correlator that operates at $128 \mathrm{MHz}$ with a power consumption of $75 \mathrm{~mW}$ is presented in [4]. An alternative approach for power consumption reduction has been introduced in [5], where power savings are achieved by reducing precision from 16-bit to a 10-bit data path, and by reducing the sampling rate from two to one sample per chip at the cost of a small performance degradation.

Another important aspect of the radio receiver design that affects its complexity is the problem of downconversion. Some relatively new work has shown that five-port technology can be used for direct conversion in SDR receivers $[6,7,8]$. This technology seems very promising because it can operate with wideband signals and over wide range of frequencies, maintaining an accurate 90 degrees phase shift. This feature makes it particularly suitable for SDR architecture, as well as for the DS-CDMA receivers that are intended for multiband operation.

In this paper we propose a new, low-complexity DSCDMA mobile terminal receiver that performs analog despreading and direct downconversion in a single stage. This has been made possible through the use of five-port device. Downconverted and despreaded signal can now be sampled at the symbol rate. In addition to lower sampling rate, digital signal processor now operates with narrowband rather than wideband signal. Further, we propose a new noncoherent 


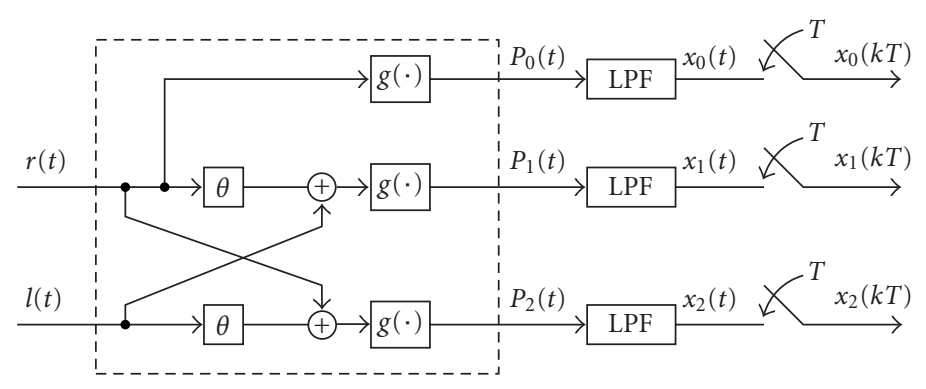

FIGURE 1: Functional block diagram of a five-port device.

analog PN code tracking scheme where the error signal that drives the tracking loop is generated by forming the absolute values (L1 norm) of the two tracking correlator outputs. It is shown that it offers similar or better performance than the well-known L2-norm-based noncoherent tracking loop.

Also, using the five-port device, we have designed a RAKE receiver that works well under wide range of conditions. Assuming that the system uses pilot signal, we introduce two solutions, depending on how the pilot signal is multiplexed. In systems that employ serially multiplexed pilot signal, we propose a RAKE structure that has a combination of fractionally and nonfractionally spaced fingers. Complexity of this receiver is low because we use already existing PN code tracking correlators to form additional fingers. For systems with parallel or continuous pilot transmission, a receiver with groups of fingers is proposed. RAKE fingers that belong to the same group have fixed spacing and are tracked with a single tracking circuitry, while the spacing between different groups depends on the multipath distribution. It is shown that in both cases performance improvement and greater robustness of the RAKE receiver can be achieved.

The paper is organized as follows. In the next section we explain the functionality of a five-port device, and show how it can be used for simultaneous downconversion and despreading in a DS-CDMA receiver. Moreover, we propose rectangular despreading that simplifies the receiver design at the cost of slight performance degradation. In Section 3, an L1-norm-based tracking loop is introduced and analyzed. Following that, two types of RAKE receiver solutions are presented in Section 4. Analysis of the five-port-based receivers is carried out in Section 5, and the power savings estimate is given in Section 6. Numerical results are provided and discussed in Section 7, followed by conclusions in Section 8.

\section{FIVE-PORT-BASED DS-CDMA RECEIVER}

The functional block diagram of a five-port device, similar to the one in [6], is shown in Figure 1. The nonlinear block $g(\cdot)$ is considered in this paper to be an ideal squaring device, which can be described as $g(x)=x^{2}$. The $\theta$ block performs phase shifting, and importantly, it maintains linearity over a wide frequency range. This is important for receivers that are intended to operate in different frequency bands. Direct conversion and despreading of the input signal $r(t)$ is performed by its addition to the local reference signal $l(t)$ and squaring the sum, followed by a subsequent filtering. In this paper we assume that technological problems associated with monolithic integration of a five-port device are not very difficult to solve, as suggested in [6].

Before describing the despreading operation, we will briefly describe how the DS-CDMA signal is generated. We consider a baseband system where $i$ th source generates binary or quaternary symbols $d_{i}(n)$ such that $\left|d_{i}(n)\right|=1$. Each symbol is spread by a PN sequence $c_{i}(k)$, such that $\left|c_{i}(k)\right|=1$, and convolved with a unit energy pulse shape $p(t)$, resulting in a baseband signal for the $i$ th user:

$$
s_{b i}(t)=\sum_{n=-\infty}^{\infty} \sqrt{E_{c}} d_{i}(n) \sum_{k=0}^{N-1} c_{i}(k) p\left(t-k T_{c}-n T\right),
$$

where $E_{c}$ is the energy per chip, $N$ is the number of chips per symbol or the spreading factor, and $T_{c}$ is the chip period. This model is applicable to any of the known CDMA modulation schemes. For example, if $d_{i}(n)$ and $c_{i}(k)$ in (1) are real binary symbols, the transmitted signal is BPSK-modulated; if only $d_{i}(n)$ is real, then we have QPSK modulation identical to the one used in IS-95, and if both $d_{i}(n)$ and $c_{i}(k)$ are complex the result is a signal with complex spreading, used in WCDMA. In this paper we assume that square-root-raised cosine pulse-shaped chips $p(t)$ are used.

Also, we will assume that the $\mathrm{PN}$ code is similar to those used in IS-95 and WCDMA, that is, it consists of a long PN code multiplied by short orthogonal codes (WalshHadamard codes) that are used for different users. While this restriction is not necessary as the receiver is designed in the same way for nonorthogonal codes, orthogonal codes are intended to eliminate the multiple access interference and can be easily implemented in the downlink.

Typically, a matched filter is used at the receiver, that is, the impulse response of the receiving filter is $p(t)$, and the resulting convolution function $R_{p p}(\tau)=\int_{-\infty}^{\infty} p(t) p(\tau-t) d t$ is a raised cosine pulse. We introduce a shorter symbolic notation for the transmitted signal:

$$
s_{b i}(t)=\sqrt{E_{c}} d_{i}(t) c_{p i}(t)
$$

where $d_{i}(t)$ and $c_{p i}(t)$ represent data and spreading code for the user $i$, respectively. The information bearing signal $s_{b i}(t)$ is modulated with a carrier signal and then transmitted. 
Due to the propagation losses, only a portion of the transmitted energy, along with the noise and interference, is collected by the antenna at the receiver. The received signal, with the carrier frequency $\omega_{c}$ and with an unknown phase $\phi$, can be expressed as

$$
r(t)=\sqrt{2} \mathfrak{R}\left\{\left[\sum_{i=0}^{K_{u}} s_{b i}(t)+n_{b}(t)\right] \mathrm{e}^{j\left(\omega_{c} t+\phi\right)}\right\},
$$

where $\mathfrak{R}[\cdot]$ indicates the real part of a complex number, $K_{u}$ is the number of active users, index $i=0$ corresponds to the pilot signal, and

$$
n_{b}(t)=n_{c}(t)+j n_{s}(t)
$$

is the complex representation of the equivalent baseband additive noise.

\subsection{Chip-matched despreading}

It will be illustrated here, based on a single-user example, that five-port technology can be used for simultaneous direct conversion and analog despreading. Instead of using two phase-shifted sinusoids for the local reference signal (to produce $I$ and Q outputs), a single direct sequence BPSK/QPSKmodulated signal is used:

$$
l(t)=\sqrt{2} \mathfrak{R}\left\{c_{p}^{*}(t) \mathbf{e}^{-j\left(\omega_{c} t-\pi / 4\right)}\right\} .
$$

Using the simple relationship for the real part of a complex number $\mathfrak{R}\{z\}=\left(z+z^{*}\right) / 2$, the result of squaring operation on the input signal becomes

$$
\begin{aligned}
P_{0}(t)=\left[\frac{1}{2} r(t)+\frac{1}{2} r^{*}(t)\right]^{2} \\
=\frac{1}{2}\left[\left(\sqrt{E_{c}} d(t) c_{p}(t)+n_{c}(t)+j n_{s}(t)\right) \mathbf{e}^{j\left(\omega_{c} t+\phi\right)}\right. \\
\left.\quad+\left(\sqrt{E_{c}} d^{*}(t) c_{p}^{*}(t)+n_{c}(t)-j n_{s}(t)\right) \mathbf{e}^{-j\left(\omega_{c} t+\phi\right)}\right]^{2} \\
=\frac{1}{2}\left[\left(\sqrt{E_{c}} d(t) c_{p}(t)+n_{c}(t)+j n_{s}(t)\right)^{2} \mathbf{e}^{j 2\left(\omega_{c} t+\phi\right)}\right. \\
\quad+\left(\sqrt{E_{c}} d^{*}(t) c_{p}^{*}(t)+n_{c}(t)-j n_{s}(t)\right)^{2} \mathbf{e}^{-j 2\left(\omega_{c} t+\phi\right)} \\
\quad+2\left(\sqrt{E_{c}} d(t) c_{p}(t)+n_{c}(t)+j n_{s}(t)\right) \\
\left.\quad+\left(\sqrt{E_{c}} d^{*}(t) c_{p}^{*}(t)+n_{c}(t)-j n_{s}(t)\right)\right] .
\end{aligned}
$$

After lowpass filtering, the output signal $x_{0}(t)$ becomes

$$
\begin{aligned}
x_{0}(t)=\operatorname{Lp}[ & \left(\sqrt{E_{c}} d(t) c_{p}(t)+n_{c}(t)+j n_{s}(t)\right) \\
& \left.\times\left(\sqrt{E_{c}} d^{*}(t) c_{p}^{*}(t)+n_{c}(t)-j n_{s}(t)\right)\right],
\end{aligned}
$$

where $\operatorname{Lp}[\cdot]$ is the notation used for lowpass filtering operation. Also, the squared sum of signal $r_{\theta}(t)+l(t)$ (the $\theta$ index notation is used to indicate phase shift) is

$$
\begin{aligned}
P_{1}(t)=\left[\frac{1}{2}\left(r_{\theta}(t)+l(t)+r_{\theta}^{*}(t)+l^{*}(t)\right)\right]^{2} \\
=\frac{1}{2}\left[2\left(\sqrt{E_{c}} d(t) c_{p}(t)+n_{c}(t)+j n_{s}(t)\right)\right. \\
\quad \times\left(\sqrt{E_{c}} d^{*}(t) c_{p}^{*}(t)+n_{c}(t)-j n_{s}(t)\right)+2\left|c_{p}(t)\right|^{2} \\
+2 c^{*}(t)\left(\sqrt{E_{c}} d(t) c_{p}(t)+n_{c}(t)+j n_{s}(t)\right) \mathrm{e}^{j(\phi+\pi / 4-\theta)} \\
\left.+2 c(t)\left(\sqrt{E_{c}} d^{*}(t) c_{p}^{*}(t)+n_{c}(t)-j n_{s}(t)\right) \mathrm{e}^{-j(\phi+\pi / 4-\theta)}\right]
\end{aligned}
$$

+ double frequency terms.

The first term in (8) is identical to the baseband portion of the $P_{0}(t)$ output, and the second term is a known constant. After lowpass filtering, and setting $\theta=\pi / 4$, the output signal $x_{1}(t)$ becomes

$$
\begin{aligned}
x_{1}(t)=\operatorname{Lp}[ & \mathfrak{R}\left\{\sqrt{E_{c}} d(t)\left|c_{p}(t)\right|^{2} \mathbf{e}^{j \phi}\right\} \\
& \left.+\mathfrak{R}\left\{c_{p}^{*}(t)\left(n_{c}(t)+j n_{s}(t)\right)\right\}\right]+x_{0}(t)+\text { const }
\end{aligned}
$$

where const is a constant due to the average power of the local oscillator. Similarly, by repeating the same procedure for $x_{2}(t)$, we get

$$
\begin{aligned}
x_{2}(t)=\operatorname{Lp}[ & -\mathfrak{J}\left\{\sqrt{E_{c}} d(t)\left|c_{p}(t)\right|^{2} \mathbf{e}^{j \phi}\right\} \\
& \left.-\mathfrak{J}\left\{c_{p}^{*}(t)\left(n_{c}(t)+j n_{s}(t)\right)\right\}\right]+x_{0}(t)+\text { const }
\end{aligned}
$$

where $\mathfrak{J}[\cdot]$ indicates the imaginary part of a complex number. The lowpass filter is used to correlate the received and local PN codes, and to remove the high-frequency components. We choose the filter constant to be $1 / \sqrt{N}$. Then, after sampling at the symbol rate, and removing the $x_{0}(n)$ and const parts, we obtain discrete variables,

$$
\begin{aligned}
& y_{1}(n)=\sqrt{N E_{c}} \mathfrak{R}\left\{d(n) \mathbf{e}^{j \phi}\right\}+\mathfrak{R}\{\nu(n)\}, \\
& y_{2}(n)=\sqrt{N E_{c}} \mathfrak{J}\left\{d(n) \mathbf{e}^{j \phi}\right\}+\mathfrak{J}\{\nu(n)\},
\end{aligned}
$$

where $\nu(n)$ is the filtered version of additive Gaussian white noise. Since $y_{1}(n)$ and $y_{2}(n)$ are real and imaginary parts of a complex value $y(n)=y_{1}(n)+j y_{2}(n)$, we can write the signal in a compact, complex form:

$$
y(n)=\sqrt{E_{s}} d(n) \mathbf{e}^{j \phi}+\nu(n)
$$


where $E_{s}=N E_{c}$ is the energy per symbol. This illustrates that the five-port device, through addition, squaring, and filtering acts as a despreader and direct converter, whose outputs are $I$ and $Q$ signal components. If it is possible to match the five-port components properly to avoid phase and gain mismatch, subtraction of the signal $x_{0}(t)$ and const dc offset from signals given in (9) and (10) can be performed before sampling, reducing the number of required A/D converters.

\subsection{Rectangular despreading}

The derivation above demonstrates that five-port technology can be used to simultaneously perform direct conversion and despreading. The resulting output signal is narrowband compared to the input signal, which makes it possible to sample it at the symbol rather faster than the chip rate. However, some important details in the receiver described above are overlooked. The local reference signal that is used as one input in the five-port circuitry is a QPSK-modulated signal. The QPSK modulation with a given pulse shape is in itself a computationally intensive operation. It involves generation of sampled version of chip pulses $p(t)$, and D/A conversion at very high speed followed by modulation with the carrier frequency. This becomes a problem, and the benefits of analog despreading disappear in a receiver where a number of different local reference signals is required to perform various tasks, such as PN code tracking, channel estimation, especially if RAKE structure is used. We now present a method that significantly reduces the complexity of local reference signal generation at the cost of only a slight performance loss. It is a simple approach, that involves using rectangular pulses $q(t)$ instead of pulses $p(t)$. The rectangular pulses $q(t)$ with the duration of $T_{c}$ are normalized to have unit energy.

Our task here is to show that the performance loss due to a nonmatched filtering approach is relatively small, while the QPSK modulation for local reference generation is greatly simplified because it can be implemented through a simple switching operation and does not require D/A converters. Following the same procedure as before, and this time including all the active users in the system, the sampled version of the output signal for user $i$ becomes

$$
\begin{aligned}
y_{i}(n)= & \frac{1}{\sqrt{N}} \int_{(n-1) T}^{n T}\left[\sum_{i^{\prime}=0}^{K_{u}} \sum_{k=0}^{N-1} d_{i^{\prime}}(n) c_{i^{\prime}}(k) p\left(t-k T_{c}-n T\right) \mathbf{e}^{j \phi}\right] \\
& \times \sum_{k=0}^{N-1} c_{i}^{*}(k) q\left(t-\tau-k T_{c}-n T\right) d t \\
& +\frac{1}{\sqrt{N}} \int_{(n-1) T}^{n T} n_{l}(t) \sum_{k=0}^{N-1} c_{i}^{*}(k) q\left(t-\tau-k T_{c}-n T\right) d t,
\end{aligned}
$$

where $\tau$ is a time delay between the received and local PN code. If we denote the result of the filtering operation, as
$R_{p q}(\tau)=\int_{-\infty}^{\infty} p(t) q(\tau-t) d t$ and derotate the signal by the angle $\phi$, the output signal becomes

$$
\begin{aligned}
z_{i}(n)=\sum_{i^{\prime}=0}^{K_{u}} \sum_{k=0}^{N-1} \sum_{m=0}^{N-1} d_{i^{\prime}}(n) c_{i^{\prime}}(k) c_{i}^{*}(m) \\
\quad \times \sqrt{\frac{E_{c}}{N}} R_{p q}\left((k-m) T_{c}+\tau\right)+v(n) \\
=d_{i}(n) \sqrt{E_{s}} R_{p q}(\tau)+I(n, \tau)+\sum_{\substack{i^{\prime}=0 \\
i^{\prime} \neq i}}^{K_{u}} M_{i^{\prime}}(n, \tau)+v(n),
\end{aligned}
$$

where $\left|c_{n}(k)\right|^{2}=1$, the self-noise term is

$$
I(n, \tau)=d_{i}(n) \sqrt{\frac{E_{c}}{N}} \sum_{k=0}^{N-1} \sum_{\substack{m \\ m \neq k}} c_{i}(k) c_{i}^{*}(m) R_{p q}\left((k-m) T_{c}+\tau\right),
$$

the multiple access interference (MAI) term from user $i^{\prime} \neq i$ is

$$
\begin{aligned}
& M_{i^{\prime}}(n, \tau)=\frac{1}{\sqrt{N}} \int_{(n-1) T}^{n T} {\left[\sum_{k=0}^{N-1} c_{i^{\prime}}(k) p\left(t-k T_{c}-n T\right) \mathbf{e}^{j \phi}\right] } \\
& \times \sum_{k=0}^{N-1} c_{i}^{*}(k) q\left(t-\tau-k T_{c}-n T\right) d t \\
&=d_{i^{\prime}}(n) \sqrt{\frac{E_{c}}{N}} \sum_{k=0}^{N-1} \sum_{\substack{m \\
m \neq k}} c_{i^{\prime}}(k) c_{i}^{*}(m) R_{p q}\left((k-m) T_{c}+\tau\right),
\end{aligned}
$$

and $\nu(n)$ is the filtered output of the thermal noise. The term associated with $m=k$ in the MAI term is equal to zero due to the assumed orthogonality of PN codes. The expressions for the self noise and MAI are also valid for the chip-matched despreading if $R_{p q}(\cdot)$ is replaced with $R_{p p}(\cdot)$.

\section{ANALOG PN CODE TRACKING}

In this section we will show how the five-port device can be used to perform the task of analog PN code tracking. We will assume that the PN code synchronization is achieved also by using the five-port device without going into the details of synchronization due to the limited space. Once the coarse PN code synchronization is achieved, that is, the local PN code is synchronized within half a chip period, the next step is the fine PN code tracking. There is a number of different tracking loops. The most commonly used tracking loop is the coherent delay-lock loop (C-DLL), which is optimal for tracking the delay difference between the acquired and the local PN code in the presence of a white Gaussian noise. However, this tracking loop does not work well in the presence of phase error. A noncoherent squaring loop, based on L2 norm (L2-DLL) is normally used to overcome this problem. 


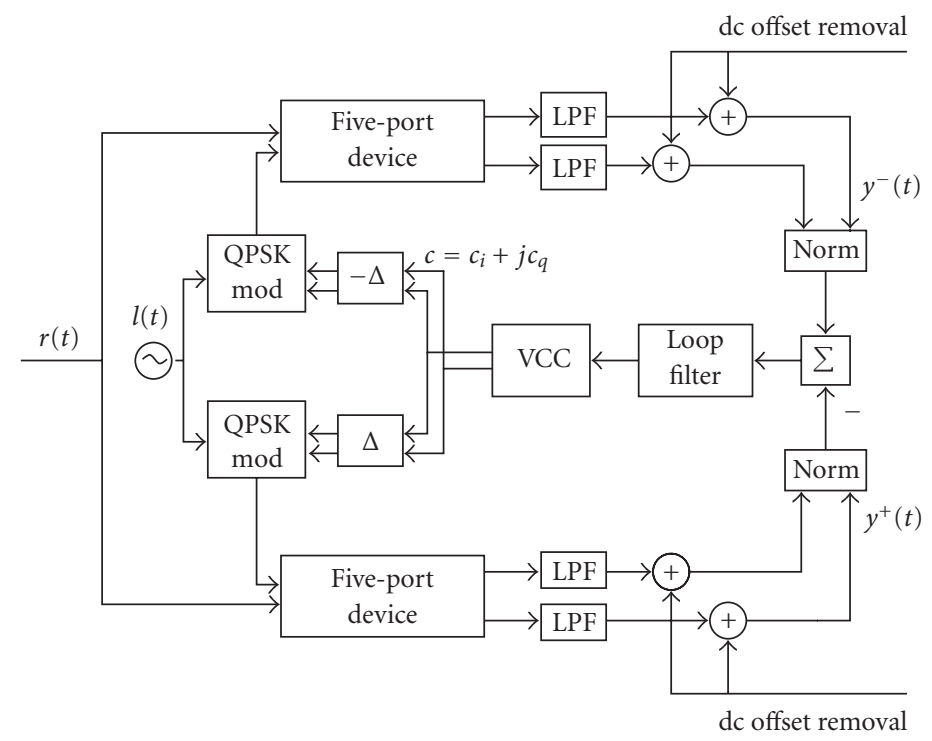

FIgUre 2: Baseband analog I-Q, early-late PN code tracking loop based on a five-port device.

Here we propose a simple to implement, noncoherent tracking scheme based on L1 instead of L2 norm, as shown in Figure 2. The idea of using L1 norm has been used in [2] for channel estimation, but not for PN code tracking. The proposed tracking loop consists of two arms, where each arm is implemented by using a five-port device, followed by the norm finding circuitry and tracking module.

The received DS-CDMA signal, assuming a single-user scenario for simplicity, has the following form:

$$
\begin{aligned}
r(t)= & \mathfrak{R}\left\{\sqrt{2 E_{c}} d(t-\tau) c_{p}(t-\tau) \mathbf{e}^{\left(j \omega_{c} t+\phi(t)\right)}\right\} \\
& +\mathfrak{R}\left\{\left[n_{c}(t)+j n_{s}(t)\right] \mathbf{e}^{j \omega_{c} t}\right\},
\end{aligned}
$$

where $\tau \leq T_{c} / 2$ is the unknown time delay at the receiver. If analog implementation is not possible because of five-port imperfections (which we did not include in this analysis), or because the pilot is serially multiplexed with the data signal, a hybrid analog/digital implementation would be required, where the outputs of early and late correlators are sampled, and the error signal forming and loop filter are implemented in digital domain. The output of a loop filter is then passed through a D/A converter to drive the voltage-controlled PN code generator (VCC). We focus our attention here on the analog implementation.

Local oscillator, QPSK-modulated with advanced and delayed versions of the PN sequence, results in local early and late reference signals:

$$
\begin{aligned}
& l_{e}(t)=\mathfrak{R}\left\{\sqrt{2} c_{q}^{*}\left(t-\hat{\tau}-\Delta T_{c}\right) \mathbf{e}^{-\left(j \omega_{c} t-\pi / 4\right)}\right\}, \\
& l_{l}(t)=\mathfrak{R}\left\{\sqrt{2} c_{q}^{*}\left(t-\hat{\tau}+\Delta T_{c}\right) \mathbf{e}^{-\left(j \omega_{c} t-\pi / 4\right)}\right\},
\end{aligned}
$$

where $\hat{\tau}$ is the estimated time delay of the received signal. The analog complex outputs of early and late arms of the tracking circuit shown in Figure 2 are

$$
y^{ \pm}(t)=\sqrt{E_{s}} R_{p q}(\varepsilon \pm \Delta) \mathbf{e}^{j \phi}+\nu^{ \pm}(t, \varepsilon, \Delta)
$$

where $\varepsilon=(\tau-\hat{\tau}) / T_{c}$ is the PN code timing error, and $\nu^{ \pm}(t, \varepsilon, \Delta)$ are the noise components in early and late branches. The error signal for L1-DLL is formed as

$$
\begin{aligned}
e_{A}(t)= & \left|\mathfrak{R}\left[y^{-}(t)\right]\right|+\left|\mathfrak{I}\left[y^{-}(t)\right]\right| \\
& -\left|\mathfrak{R}\left[y^{+}(t)\right]\right|-\left|\mathfrak{I}\left[y^{+}(t)\right]\right| \\
= & \sqrt{E_{s}} S_{A}(\varepsilon, \Delta ; \phi)+w_{A}(t),
\end{aligned}
$$

where

$$
\begin{aligned}
S_{A}(\varepsilon, \Delta ; \phi) & =\frac{1}{\sqrt{E_{s}}} E\left[e_{A}(t) \mid \phi\right] \\
& =\frac{1}{\sqrt{E_{s}}}\left[M_{I}^{-}+M_{Q}^{-}-M_{I}^{+}-M_{Q}^{+}\right]
\end{aligned}
$$

is the average tracking curve conditioned under $\phi$, with

$$
\begin{aligned}
& M_{I}^{ \pm}=\sigma_{n} \sqrt{\frac{2}{\pi}} \mathbf{e}^{-\left(m_{I}^{ \pm}\right)^{2} / 2 \sigma_{n}^{2}}+\left|m_{I}^{ \pm}\right| \operatorname{erf}\left(\frac{\left|m_{I}^{ \pm}\right|}{2 \sigma_{n}^{2}}\right), \\
& M_{Q}^{ \pm}=\sigma_{n} \sqrt{\frac{2}{\pi}} \mathbf{e}^{-\left(m_{Q}^{ \pm}\right)^{2} / 2 \sigma_{n}^{2}}+\left|m_{Q}^{ \pm}\right| \operatorname{erf}\left(\frac{\left|m_{Q}^{ \pm}\right|}{2 \sigma_{n}^{2}}\right),
\end{aligned}
$$

where $\sigma_{n}^{2}=N_{0} / 2$ and

$$
\begin{aligned}
& m_{I}^{ \pm}=\sqrt{E_{s}} R_{p q}(\varepsilon \pm \Delta) \cos \phi, \\
& m_{Q}^{ \pm}=\sqrt{E_{s}} R_{p q}(\varepsilon \pm \Delta) \sin \phi .
\end{aligned}
$$


The noise term is

$$
w_{A}(t)=e_{A}(t)-E\left[e_{A}(t)\right]
$$

The standard approach of DLL circuitry analysis that consists of finding the noise autocorrelation function first, and then its power spectral density, is difficult to carry out in this case because the absolute values are involved. To avoid this difficulty, an easier approach has been derived that produces results that are in relatively good agreement with simulations. As pointed out in [9], performance of analog DLL is similar to the performance of digital DLL. For digital DLL it is much easier to find the noise power spectral density at $f=0$, and then use its scaled version to approximate power spectral density of the analog noise process. The scaling factor is the symbol period $T$. Therefore, we write the noise power spectral density of the equivalent digital L1-DLL as

$$
\S_{W_{A}}(f=0, \varepsilon=0)=V_{I}^{-}+V_{Q}^{-}+V_{I}^{+}+V_{Q}^{+},
$$

where

$$
\begin{aligned}
& V_{I}^{ \pm}=\sigma_{n}^{2}+\left(m_{I}^{ \pm}\right)^{2}-\left(M_{I}^{ \pm}\right)^{2}, \\
& V_{Q}^{ \pm}=\sigma_{n}^{2}+\left(m_{\bar{Q}}^{ \pm}\right)^{2}-\left(M_{\bar{Q}}^{ \pm}\right)^{2} .
\end{aligned}
$$

If the signal-to-noise ratio is not too low, DLL circuits can be analyzed by the approximate linear model. If we denote the " $S$ " curve slope as

$$
\eta_{A}=\left.\frac{\partial S_{A}(\varepsilon)}{\partial \varepsilon}\right|_{\varepsilon=0}
$$

then, following a procedure outlined in [10], the variance of the timing error is

$$
\sigma_{\varepsilon_{A}}^{2}=\frac{\S_{W_{A}}(0,0) T}{E_{s} \eta_{A}^{2}} \int_{-\infty}^{\infty}|H(2 \pi f j)|^{2} d f=\frac{2 \delta_{W_{A}}(0,0) B_{L} T}{E_{s} \eta_{A}^{2}},
$$

where

$$
B_{L}=\frac{1}{2} \int_{-\infty}^{\infty}|H(2 \pi f j)|^{2} d f
$$

is the loop filter bandwidth, and the closed loop transfer function is defined as

$$
H(s)=\frac{\sqrt{E_{s}} \eta_{A} K F(s)}{s+\sqrt{E_{s}} \eta_{A} K F(s)}
$$

where $F(s)$ is the transfer function of the loop filter, and $K$ is the gain of the VCC. The tracking error variance for the L1-DLL in (28) is conditioned under $\phi$, and the average jitter can be determined as

$$
\sigma_{\varepsilon_{A}}^{2}=\int_{\phi} \sigma_{\varepsilon_{A} \mid \phi}^{2} p(\phi) d \phi
$$

If we define signal-to-noise ratio of the noise loop as $\gamma_{D}=E_{s} /\left(N_{0} B_{L} T\right)$, we can compare the RMS jitter of the L1DLL with the RMS jitter of coherent and L2-DLL (noncoherent) tracking circuitries. The tracking error variance for the coherent DLL is [11]

$$
\sigma_{\varepsilon_{c}}^{2}=\frac{2\left[R_{p q}(0)-R_{p q}(2 \Delta)\right]}{\gamma_{D} \eta_{C}^{2}},
$$

and for the L2-DLL the tracking error variance is [10]

$$
\sigma_{\varepsilon_{N}}^{2}=\frac{2\left[R_{p q}(0)-R_{p q}(2 \Delta)\right]}{\gamma_{D} \eta_{C}^{2} \rho_{N}}
$$

where $\rho_{N}$ represents the noncoherent loss for L2-DLL given as

$$
\rho_{N}=\frac{\eta_{N}^{2}}{\eta_{C}^{2}} \frac{\gamma_{D} B_{L} T}{(4 / 3)\left[R_{p q}(0)+R_{p q}(2 \Delta)\right]+4 \gamma_{D} B_{L} T R_{p q}^{2}(\Delta)} .
$$

\section{FIVE-PORT-DEVICE-BASED RAKE RECEIVERS}

So far we have seen that it is possible to design a DS-CDMA receiver with analog despreading and direct conversion using five-port device. The same approach can be used in a design of RAKE receivers. RAKE receivers consist of a number of correlators, where the local reference for each correlator is generated with a different PN code offset. Different PN code delays are locked to different times of arrival of multipath components (time-delayed replicas of the same signals due to reflection) of the received signal. The weighted sum of the correlator outputs is formed to produce a decision variable, where the combining coefficients are selected such that the decision variable has maximal SNR. Different multipath components can fade independently, and their combination at the output of a RAKE receiver results in a smaller variation of the SNR, which in turn improves the performance of the receiver. The desirable property of the propagation channel is that the delay spread extends over several chip period intervals, and that each correlator output is independent of the other. This would make the task of tracking of multipath components that fade independently much easier. However, the independence between two relatively closely spaced correlators (in the case of RAKE receiver the correlators are called fingers) does not always exist. The correlation between the multipath components with a relatively close spacing can be modelled with a discrete set of multipath components that are separated by less than one chip period in time. This scenario is known as nonresolvable ${ }^{1}$ multipath, and it can have detrimental effect on the receiver performance $[12,13]$. This is particularly true in the case of fast fading, where channel changes faster than the averaging time of the loop filter in the tracking module. In this case, the loop filter averages out

\footnotetext{
${ }^{1}$ Resolvable multipath refers to multipath components that are separated by one or more than one chip period apart.
} 
the fading signal. Then, the average instead of instantaneous fading signal determines the tracking point. As a result, a tracking bias occurs, which leads to a significant performance degradation of the RAKE receiver.

Having this in mind, our goal is to design a low complexity, low-power consumption RAKE receiver that works well under a wide range of circumstances. Assuming that DSCDMA systems use a pilot signal in the forward link, we introduce two solutions, depending on how the pilot signal is multiplexed. In systems that employ serially multiplexed pilot signal, we propose a RAKE structure that has a combination of fractionally (less than one chip period) and nonfractionally (more or equal to the one chip period) spaced fingers. Complexity of this receiver is low because, as we will see, we can use already existing PN code tracking correlators to form additional fingers. For systems with parallel or continuous pilot transmission, a receiver with groups of fingers is proposed. RAKE fingers that belong to the same group have fixed spacing and are tracked with a single tracking loop, while the spacing between different groups depends on the multipath distribution. It is shown that in both cases performance improvement and greater robustness of the RAKE receiver can be achieved.

The two proposed solutions do not have to be necessarily tied to the two previously mentioned types of pilot signal transmission. Both of these solutions can be applied to the receiver that operates under any type of pilot transmission, and even to receivers used in a system without a pilot signal. However, from the complexity point of view, it seems reasonable to classify them in the aforementioned way.

\subsection{RAKE receiver with fractional finger spacing}

If the pilot is serially multiplexed with the data channel, the PN code tracking of the five-port-based receiver can be implemented digitally to achieve better performance. In this case, we need more A/D converters than in the case of analog PN code tracking, but the same correlators can be used for RAKE combining during the data transmission. We will show that it is possible to improve the performance of the RAKE receiver with a small number of fingers, by converting each conventional RAKE finger into three fractionally spaced fingers without changing the hardware structure of the receiver. The idea of using fractionally spaced fingers has already been suggested in [14], where it is shown that the performance of the RAKE receiver in the presence of signal correlation can be improved by using fractional chip finger spacing. Instead of using optimal fractional finger spacing, that may be hard to calculate at the receiver because it depends on the channel, our solution uses fixed, half-chip finger spacing. Though not optimal, this solution leads to an improved receiver performance. The so-called early and late correlators that are used in the proposed receiver already exist in the receiver and are part of the PN code tracking circuitry. We will also investigate a very simple receiver structure [15], where only early and late correlator outputs are used in the combining process, and we will call it an early-late combiner (ELC). It will be shown that even without using the on-time correlator, there is a performance gain under fast fading, but this

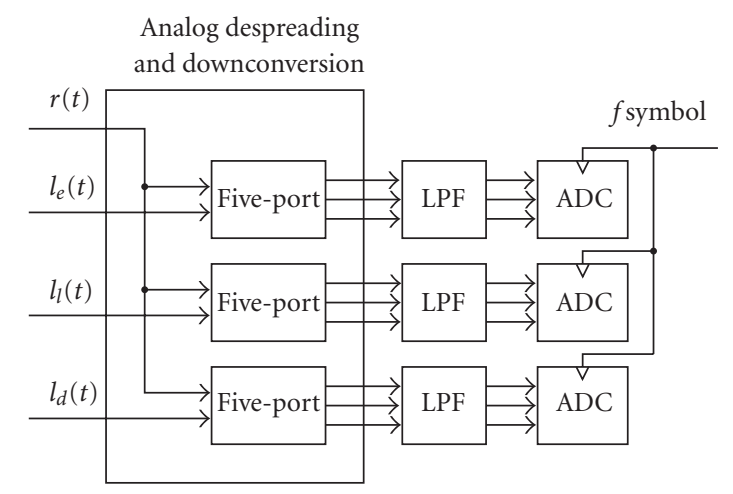

FIgUre 3: One finger of a RAKE receiver.

improvement disappears under slow fading. The proposed receiver with fractionally spaced fingers is identified as earlyon-late combiner (EOLC), because it uses early and late correlators like the previous one and on-time correlator in the process of combining. Conventional RAKE receiver consists of on-time correlator only for each finger.

One finger of the conventional RAKE receiver based on a five-port device, that will be transformed either in two (ELC receiver), or three (EOLC receiver) fingers, is shown in Figure 3. Local reference signal $l_{e}(t)$ for early correlation, $l_{d}(t)$ and $l_{l}(t)$ for late correlation are generated by multiplying the carrier frequency with rectangular pulses $q(t)$ through the switching operation.

\subsection{RAKE receiver with groups of fingers}

When the pilot is continuously transmitted, the five-portbased receiver can perform PN code tracking in analog domain. However, even with such low complexity, if we try to implement a conventional RAKE receiver, each finger would require a separate tracking circuitry. Therefore, such implementation would result in a replication of too many components. A new solution is introduced here, where a number of uniformly spaced fingers are added, but the number of tracking circuitries is kept low. The PN codes for these new correlators are delayed or advanced by one chip period with respect to the existing on-time correlator, resulting in a group of uniformly spaced fingers. Importantly, each group of fingers formed in this way shares one tracking circuitry, that is, the tracking is the same as for the conventional RAKE receiver. Normally, dominant multipath components are not isolated, and it is possible to pick up some energy from the surrounding components even without a need to track them. The proposed receiver consists of a relatively small number of groups of RAKE fingers, as shown in Figure 4.

A block diagram of one group of the proposed receiver that consists of three correlators is shown in Figure 5.

\section{PERFORMANCE ANALYSIS}

In the analysis of the proposed RAKE receivers we will use a discrete Rayleigh fading channel model. Multipath components are grouped together in resolvable clusters, where each cluster consists of unresolvable components. In a system with 


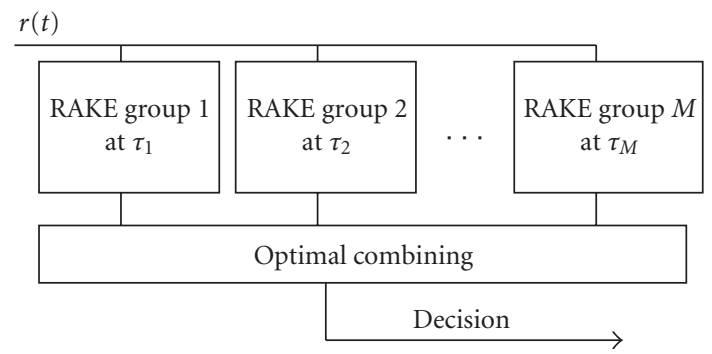

FIGURE 4: RAKE receiver with groups of equally spaced fingers.

$K_{u}$ active users, we will denote by $r_{i}(t)$ the signal intended for user $i$. Then, the composite signal at the input of the receiver can be written as

$$
r(t)=\sum_{l=1}^{L} \sum_{u_{l}=1}^{U_{l}} \sum_{i=0}^{K_{u}} a_{u_{l}}(t) r_{i}\left(t-\tau_{u_{l}}\right)
$$

where $L$ is the number of resolvable multipath clusters, $U_{l}$ is the number of unresolvable components within the $l$ th multipath cluster, $a_{u_{l}}(t)$ are independent complex Gaussian random processes used to represent Rayleigh fading, and index $i=0$ corresponds to the pilot signal. Since we are considering downlink reception, it should be noted that pilot and all data signals are subjected to the same channel.

The result of despreading of the $l$ th cluster and sampling every $T$ seconds at the $i$ th user receiver can be written as

$$
\begin{aligned}
y_{i l}\left(n, \hat{\tau}_{l}\right) & =\frac{1}{\sqrt{N}} \int_{-\infty}^{\infty} r(t) c_{q i}^{*}\left(t-\hat{\tau}_{l}\right) \mathbf{e}^{-j \omega_{c} t} d t \\
& =s_{l}\left(n, \hat{\tau}_{l}\right)+I\left(\hat{\tau}_{l}\right)+P\left(\hat{\tau}_{l}\right)+\sum_{\substack{i^{\prime} \\
i^{\prime} \neq i}} M_{i^{\prime} l}\left(n, \hat{\tau}_{l}\right)+v(n),
\end{aligned}
$$

where $c_{q i}(t)$ is the local PN code reference, $N$ is the number of chips per symbol, and

$$
s_{l}\left(n, \hat{\tau}_{l}\right)=\sum_{u_{l}=1}^{U_{l}} \sqrt{E_{s}} a_{u_{l}}(t) d_{i}(n) R_{p q}\left(\hat{\tau}_{l}-\tau_{u_{l}}\right)
$$

is the desired signal component. The noise components are decomposed in the self-noise part

$$
\begin{array}{r}
I\left(\hat{\tau}_{l}\right)=\frac{1}{\sqrt{N}} \sum_{u_{l}=1}^{U_{l}} \sum_{\substack{r=1 \\
r \neq s}}^{N} \sum_{s=1}^{N} \sqrt{E_{c}} a_{u_{l}}(t) d_{i}(n) c_{i}(r) c_{i}^{*}(s) \\
\times R_{p q}\left((r-s) T_{c}+\hat{\tau}_{l}-\tau_{u_{l}}\right),
\end{array}
$$

interpath interference from the other multipath clusters:

$$
P\left(\widehat{\tau}_{l}\right)=\frac{1}{\sqrt{N}} \int_{-\infty}^{\infty} \sum_{\substack{l^{\prime}=1 \\ l^{\prime} \neq l}}^{L} \sum_{u_{l}^{\prime}=1}^{U_{l^{\prime}}} \sqrt{E_{c}} a_{u_{l^{\prime}}}(t) d_{i}(t) c_{p i}\left(t-\tau_{u_{l^{\prime}}}\right) c_{q i}^{*}\left(t-\widehat{\tau}_{l}\right) d t,
$$

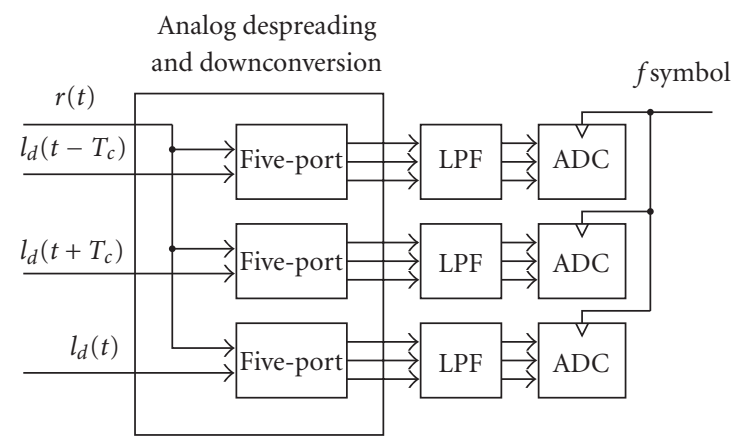

FIgURE 5: The $m$ th group for the proposed LORC RAKE receiver.

contribution from an unwanted user $i^{\prime}$, or the MAI term

$$
\begin{gathered}
M_{i^{\prime} l}\left(n, \hat{\tau}_{l}\right)=\frac{1}{\sqrt{N}} \int_{-\infty}^{\infty} \sum_{l=1}^{L} \sum_{u_{l}=1}^{U_{l}} \sqrt{E_{c}} a_{u_{l}}(t) d_{i^{\prime}}(t) c_{p i^{\prime}}\left(t-\tau_{u_{l}}\right) \\
\times c_{q i}^{*}\left(t-\hat{\tau}_{l}\right) d t,
\end{gathered}
$$

and $\nu(n)$ is the filtered version of additive Gaussian noise.

To account for the effect of each of these components on the decision variable, we derive their second-order statistics. Each of the noise components has zero mean.

\subsection{Self-noise}

If the self-noise is treated as a zero mean random process, then the correlation function of its real part is defined as

$$
R_{I}\left(\widehat{\tau}_{l}, \Delta \tau\right)=E\left[\mathfrak{R}\left[I\left(\hat{\tau}_{l}\right)\right] \mathfrak{R}\left[I\left(\widehat{\tau}_{l}+\Delta \tau\right)\right]\right] .
$$

After a quite involved random variable exercise, the correlation function conditioned on the multipath is found to be

$$
\begin{array}{r}
R_{I}\left(\hat{\tau}_{l}, \Delta \tau\right)= \\
\frac{E_{s}}{2 N} \Re\left\{\sum _ { u _ { l } = 1 } ^ { U _ { l } } \sum _ { v _ { l } = 1 } ^ { U _ { l } } \left[a_{u_{l}} a_{v_{l}}^{*} \sum_{m \neq 0} R_{p q}\left(m T_{c}+\hat{\tau}_{l}-\tau_{u_{l}}\right)\right.\right. \\
\times R_{p q}\left(m T_{c}+\hat{\tau}_{l}+\Delta \tau-\tau_{v_{l}}\right) \\
+a_{u_{l}} a_{v_{l}} \sum_{m \neq 0} R_{p q}\left(m T_{c}+\hat{\tau}_{l}-\tau_{u_{l}}\right) \\
\left.\left.\times R_{p q}\left(\hat{\tau}_{l}+\Delta \tau-\tau_{v_{l}}-m T_{c}\right)\right]\right\},
\end{array}
$$

where $E_{s}=N E_{c}$ is energy per symbol, and $E_{c}$ is the energy per transmitted chip.

The self-noise contribution to the noise correlation matrix for fractionally spaced fingers can be directly calculated from the above correlation function. Also, for $\Delta \tau=0$ the variance of the self-noise at the finger position $\hat{\tau}_{l}$ can be 
found. Similarly, the noise variance for the simple one-finger receiver and without fading can be calculated as

$$
V_{I}=R_{I}(\tau, 0)=\frac{1}{2} \frac{E_{s}}{N} l_{p q}(\tau)
$$

where

$$
\iota_{p q}(\tau)=\sum_{m \neq 0}\left[R_{p q}^{2}\left(m T_{c}+\tau\right)+R_{p q}\left(m T_{c}+\tau\right) R_{p q}\left(m T_{c}-\tau\right)\right] .
$$

Clearly, if $q(t)=p(t)$, the self-noise component disappears for $\tau=0$, that is, $\iota_{p q}(0)=0$, because $R_{p q}\left(m T_{c}\right)=0$ for $m \neq 0$.

\subsection{Interpath interference}

For the PN code offset larger than one chip period $T_{c}$, the interpath interference (IPI) from the desired user, as well as from the other users, acts like the multiple access interference from the other users in a nonorthogonal CDMA system (e.g., reverse link). The interpath interference, given above, can be written as

$$
\begin{gathered}
P_{p}\left(n, \hat{\tau}_{l}\right) \approx \frac{1}{\sqrt{N}} \sum_{\substack{l^{\prime}=1 \\
l^{\prime} \neq l}}^{L} \sum_{u_{l}^{\prime}=1}^{U_{l^{\prime}}} \sqrt{E_{c}} a_{u_{l^{\prime}}} d_{i}(n) \sum_{r=1}^{N} c_{i^{\prime}}(r) c_{i}^{*}(r) \\
\times R_{p q}\left(\hat{\tau}_{l}-\tau_{u_{l^{\prime}}}\right) d t
\end{gathered}
$$

where the approximation comes from the fact that the cross terms that come with $r \neq s$ are much smaller than the terms that come with $r=s$. By following the same procedure as for the self-noise, the correlation function is

$$
\begin{array}{r}
R_{P}\left(\hat{\tau}_{l}, \Delta \tau\right)=\frac{E_{s}}{2 N} \mathfrak{R}\left\{\sum_{\substack{l^{\prime}=1 \\
l^{\prime} \neq l}}^{L} \sum_{u_{l^{\prime}}=1}^{U_{l}} \sum_{v_{l^{\prime}}=1}^{U_{l}} a_{u_{l^{\prime}}} a_{v_{l^{\prime}}}^{*} R_{p q}\left(\hat{\tau}_{l}-\tau_{u_{l^{\prime}}}\right)\right. \\
\left.\times R_{p q}\left(\hat{\tau}_{l}+\Delta \tau-\tau_{v_{l^{\prime}}}\right)\right\} .
\end{array}
$$

The IPI variance, obtained from the expression above after setting $\Delta \tau=0$, changes together with the change in the signal power, which makes the analysis of our receiver very complicated. To avoid this, we can use the average IPI power. Also, it is possible to remove the effect of the IPI if the multipath component delays are known [16].

After removing the conditioning on the multipath, and setting $\Delta \tau=0$, and $E_{s}=2 E_{b}$, the average IPI variance is

$$
V_{P}=\frac{E_{b}}{N} \sum_{\substack{l^{\prime}=1 \\ l^{\prime} \neq l}}^{L} \sum_{u_{l^{\prime}}=1}^{U_{l}}\left|a_{u_{l^{\prime}}}\right|^{2} R_{p q}^{2}\left(\hat{\tau}_{l}-\tau_{u_{l^{\prime}}}\right)
$$

This expression is similar to the one [17] for the MAI terms in nonorthogonal CDMA systems. The IPI term from the pilot signal exists for parallel multiplexed pilot.

\subsection{MAl from the same multipath cluster}

To analyze the effects of multiple access interference, it is sufficient to analyze the effects of a single MAI user, because the interference from each user will be mutually independent, and thus can be added together. The expression for the MAI contribution of an unwanted $i^{\prime}$ th user in the expanded form is

$$
\begin{aligned}
& M_{i^{\prime} l}\left(n, \hat{\tau}_{l}\right)= \\
& \begin{aligned}
\frac{1}{\sqrt{N}} \sum_{u_{l}=1}^{U_{l}} \sum_{\substack{r=1 \\
r \neq s}}^{N} \sum_{s=1}^{N} \sqrt{E_{c}} a_{u_{l}}(t) d_{i}(n) c_{i^{\prime}}(r) c_{i}^{*}(s) \\
\quad \times R_{p q}\left((r-s) T_{c}+\hat{\tau}_{l}-\tau_{u_{l}}\right)+P_{p l^{\prime}}\left(\hat{\tau}_{l}\right),
\end{aligned}
\end{aligned}
$$

where $P_{p l^{\prime}}\left(\hat{\tau}_{l}^{\prime}\right)$ is the interpath interference from the MAI, and we note that the sum of terms in (48) for $r=s$ is equal 0 , which is a consequence of downlink PN code orthogonality between different users. The MAI correlation function is

$$
\begin{aligned}
& R_{M}\left(\hat{\tau}_{l}, \Delta \tau\right)= \\
& \frac{E_{s}}{2 N} \mathfrak{R}\left\{\sum_{u_{l}=1}^{U_{l}} \sum_{v_{l}=1}^{U_{l}} a_{u_{l}} a_{v_{l}}^{*} \sum_{m \neq 0} R_{p q}\left(m T_{c}+\hat{\tau}_{l}-\tau_{u_{l}}\right)\right. \\
& \left.\times R_{p q}\left(m T_{c}+\hat{\tau}_{l}+\Delta \tau-\tau_{v_{l}}\right)\right\}
\end{aligned}
$$

which is an expression similar to the one obtained for the self-noise.

For the single-finger receiver and with no fading, the MAI variance is

$$
V_{M}=R_{M}(\tau, 0)=\frac{1}{2} \frac{E_{s}}{N} \mu_{p q}(\tau)
$$

where

$\mu_{p q}(\tau)=\frac{1}{N} \sum_{m \neq 0} \sum_{\substack{q \\ q \neq p}} R_{p q}^{2}\left((p-q) T_{c}+\tau\right)=\sum_{m \neq 0} R_{p q}^{2}\left(m T_{c}+\tau\right)$.

Again, if $q(t)=p(t)$, the MAI components vanish for $\tau=0$, that is, $\mu_{p q}(0)=0$, because $R_{p q}\left(m T_{c}\right)=0$ for $m \neq 0$, which is a direct consequence of orthogonality between the PN codes used in the forward link.

\subsection{Additive noise correlation}

The real and imaginary parts of the additive white noise term have the correlation function

$$
R_{\nu}\left(\hat{\tau}_{l}, \Delta \tau\right)=\frac{N_{0}}{2} R_{q q}(\Delta \tau)
$$




\subsection{Total noise correlation}

The total noise correlation function can now be obtained by adding all of the previously derived correlations:

$$
\begin{aligned}
R_{w}\left(\hat{\tau}_{l}, \Delta \tau\right) & =R_{I}\left(\hat{\tau}_{l}, \Delta \tau\right)+R_{P}\left(\hat{\tau}_{l}, \Delta \tau\right)+R_{M}\left(\hat{\tau}_{l}, \Delta \tau\right)+R_{\nu}\left(\hat{\tau}_{l}, \Delta \tau\right) \\
& =\frac{N_{0}}{2} R_{q q}(\Delta \tau)+K_{u} V_{P} R_{p q}(\Delta \tau)+\varepsilon \delta(\Delta \tau) \\
& \approx \frac{I_{0}}{2} R_{q q}(\Delta \tau)+V_{I M} \delta(\Delta \tau),
\end{aligned}
$$

where $K_{u}$ is the number of users that are occupying the channel, $I_{0}$ is the effective noise power spectral density, and $V_{I M}=$ $V_{I}+V_{M}$, where $V_{I}$ and $V_{M}$ are self-noise and MAI variances, approximately takes into account the effects of self-noise and MAI from the same cluster. For typical SNR values and for high processing gain, its value will be well below the $I_{0}$ level.

\subsection{Single-finger uncoded bit error rate}

The single-finger receiver signal output without fading is given in (14). The decision variable is based on the real or both real and imaginary parts, depending on the modulation scheme. Since the imaginary part has the same statistics as the real part, we will focus on the real part only. Statistics of the decision variable are determined by its mean and variance. The mean and the variance of the real part of the decision variable are

$$
\begin{aligned}
E\left[\Re\left\{z_{i}(n)\right\}\right] & = \pm \sqrt{E_{b}} R_{p q}(\tau), \\
\operatorname{Var}[\Re\{z(n)\}] & =V_{I}+V_{M}+V_{N},
\end{aligned}
$$

where $V_{I}$ and $V_{M}=\sum_{\substack{i^{\prime}=0 \\ i^{\prime} \neq i}} V_{M_{i^{\prime}}}$ are previously determined variances for the self-noise and MAI, and

$$
V_{N}=\frac{1}{2} \sigma_{\nu}^{2}(n)=\frac{1}{2} 0.903 N_{0}
$$

is the variance of the real part of the thermal noise, as determined in Appendix A.

The noise variance due to the self-noise and MAI depends on the modulation scheme. For instance, $E_{s}=E_{b}$ for IS-95 type of modulation, and $E_{s}=2 E_{b}$ for WCDMA. Also, noise statistics for BPSK-modulated direct sequence signal will be identical to ones for WCDMA. For example, the selfnoise variance for BPSK and WCDMA is two times larger than the self-noise variance for the IS-95. The same result has been obtained through a different derivation process in [17]. This also applies for the MAI variance.

Finally, the SNR per bit [18] of the sampled signal at the output of the five-port device becomes

$$
\gamma=\frac{1}{2} \frac{\left[E\left[\Re\left\{z_{i}(n)\right\}\right]\right]^{2}}{\operatorname{Var}\left[\mathfrak{R}\left\{z_{i}(n)\right\}\right]}=\frac{E_{b}}{N_{0}} \xi_{p q}(\tau),
$$

where

$$
\xi_{p q}(\tau)=\frac{R_{p q}^{2}(\tau)}{0.903+(2 / N)\left(E_{b} / N_{0}\right) \iota_{p q}(\tau)+(2 / N)\left(E_{b} / N_{0}\right) K_{u} \mu_{p q}(\tau)}
$$

is the SNR loss due to the self-noise, MAI, and PN code tracking error $\tau$. The uncoded bit error rate for a given tracking error $\tau$ can be calculated as [17]

$$
P_{e}(\tau)=\frac{1}{2} \operatorname{erfc}(\sqrt{\gamma})=\frac{1}{2} \operatorname{erfc}\left(\sqrt{\frac{E_{b}}{N_{0}} \xi(\tau)}\right)
$$

\subsection{Uncoded bit error rate for RAKE receivers}

We will determine uncoded bit error rates for the proposed RAKE receivers and compare them with the conventional RAKE receiver. We start with introducing the signal and noise vectors for the receivers in question. The RAKE receiver signal, before combining, can be written in a complex vector form as

$$
\mathbf{y}_{x}=\mathbf{s}_{x}+\mathbf{w}_{x}
$$

where $\mathbf{y}_{x}$ is the vector whose elements are correlator outputs $y_{l}\left(\hat{\tau}_{l}\right)$ given in (36), the subscript $x$ will be replaced with the corresponding initials for each receiver, and $\mathbf{w}_{x}$ is the noise vector that includes the effects of additive Gaussian noise, self-noise, IPI, and MAI. For the conventional RAKE receiver with $L$ fingers, the signal vector is

$$
\mathbf{s}_{C}=\left[s_{1}\left(\hat{\tau}_{1}\right) s_{2}\left(\hat{\tau}_{2}\right) \cdots s_{L}\left(\hat{\tau}_{L}\right)\right]^{T},
$$

where $s_{l}\left(\hat{\tau}_{l}\right)$ are given in (37). Similarly, the noise vector is

$$
\mathbf{w}_{C}=\left[w_{1}\left(\hat{\tau}_{1}\right) w_{2}\left(\hat{\tau}_{2}\right) \cdots w_{L}\left(\hat{\tau}_{L}\right)\right]^{T}
$$

The ELC combining RAKE receiver has the signal vector given by

$$
\begin{aligned}
& \mathbf{s}_{\mathrm{ELC}} \\
& \quad=\left[s_{1 E}\left(\hat{\tau}_{1}-\Delta T_{c}\right) s_{1 L}\left(\hat{\tau}_{1}+\Delta T_{c}\right) \cdots s_{L E}\left(\hat{\tau}_{L}-\Delta T_{c}\right) s_{L L}\left(\hat{\tau}_{L}+\Delta T_{c}\right)\right]^{T},
\end{aligned}
$$

and noise vector is formed in the similar way. The EOLC combiner has the signal vector

$$
\begin{aligned}
\mathbf{s}_{\mathrm{EOLC}}=[ & s_{1 L}\left(\hat{\tau}_{1}-\Delta T_{c}\right) s_{1 O}\left(\hat{\tau}_{1}\right) s_{1 R}\left(\hat{\tau}_{1}+\Delta T_{c}\right) \\
& \left.\cdots s_{L L}\left(\hat{\tau}_{L}-\Delta T_{c}\right) s_{L O}\left(\hat{\tau}_{L}\right) s_{L R}\left(\hat{\tau}_{L}+\Delta T_{c}\right)\right]^{T},
\end{aligned}
$$

where $\Delta=0.5$. The RAKE receiver with groups of fingers, that we will call LORC, has the same vector form as the EOLC receiver, with the difference that $\Delta=1$. 
The elements of the noise covariance matrix, $\mathbf{R}_{w_{x}}=$ $E\left[\mathbf{w}_{x} \mathbf{w}_{x}^{H}\right]$, are formed from the correlation function defined in Section 2. Due to the fractional finger spacing for the EOLC receiver, the noise covariance matrix is not an identity matrix. Thus, to ensure that noise is not correlated, combining process is preceded with the noise decorrelation or whitening. Since noise covariance matrix is positive definite, a positive definite square root matrix $\mathbf{R}_{w_{x}}^{1 / 2}$ exists [19], such that $\mathbf{R}_{w_{x}}=\mathbf{R}_{w_{x}}^{1 / 2} \mathbf{R}_{w_{x}}^{1 / 2}$, where

$$
\mathbf{R}_{w_{x}}^{1 / 2}=\mathbf{U}_{w_{x}} \Lambda_{w_{x}}^{1 / 2} \mathbf{U}_{w_{x}}^{H}
$$

for the unitary decomposition $\mathbf{R}_{w_{x}}=\mathbf{U}_{w_{x}} \boldsymbol{\Lambda}_{w_{x}} \mathbf{U}_{w_{x}}^{H}$. Based on this, we can choose a noise decorrelation matrix to be

$$
\mathbf{D}=\mathbf{R}_{w_{x}}^{-1 / 2}=\mathbf{U}_{w_{x}} \boldsymbol{\Lambda}_{w_{x}}^{-1 / 2} \mathbf{U}_{w_{x}}^{H},
$$

because

$$
E\left[\mathbf{D w}(\mathbf{D w})^{H}\right]=\mathbf{I} .
$$

The received signal, after noise decorrelation and before combining has the following form:

$$
\mathrm{Dy}_{x}=\mathrm{D} \mathbf{s}_{x}+\mathrm{Dw}_{x}
$$

and the optimal RAKE combining coefficients are then $\boldsymbol{\alpha}=$ $\left(\mathbf{D} \mathbf{s}_{x}\right)^{H}$. Then, the SNR per bit at the output of a combiner is given by the quadratic form

$$
\gamma_{x}=\frac{E_{b}}{I_{0}} \mathbf{s}_{x}{ }^{H} \mathbf{R}_{w_{x}}^{-1} \mathbf{s}_{x}
$$

which can, through a series of matrix transformations, be brought into another quadratic form, as shown in the appendix:

$$
\gamma_{x}=\frac{E_{b}}{I_{0}} \sum_{i}\left|u_{x, i}\right|^{2} \lambda_{x, i}=\sum_{i}\left|u_{x, i}\right|^{2} \gamma_{x, i}
$$

where $\left|u_{x, i}\right|^{2} \gamma_{x, i}$ are $\chi^{2}$ distributed independent random variables. While the process of arriving at the expression (69) for fractionally and nonfractionally spaced fingers is slightly different, the final expression is the same. Clearly, after the quadratic form transformation, the SNR has become a wellknown linear combination of independent central $\chi^{2}$ random variables. Thus, the pdf for $\gamma_{x}$ can be found by using the characteristic function approach. It is convenient to use Laplace transform, since $\lambda_{x, i}$ are real positive constants. The pdf can be found directly by finding the inverse Laplace transform of the characteristic function for the case when the eigenvalues $\lambda_{x, i}$ are distinct:

$$
p_{x}\left(\gamma_{x}\right)=\mathcal{L}^{-1}\left[\prod_{i=1}^{P_{x}} \frac{1}{1+s \gamma_{x, i}}\right]=\sum_{i=1}^{P_{x}} \frac{A_{i}}{\gamma_{x, i}} e^{-\gamma / \gamma_{x, i}},
$$

where

$$
A_{i}=\prod_{\substack{j=1 \\ j \neq i}}^{P_{x}} \frac{\lambda_{x, i}}{\lambda_{x, i}-\lambda_{x, j}}
$$

and $P_{x}=L$ for the conventional RAKE receiver, $P_{x}=2 L$ for the ELC receiver, and $P_{x}=3 L$ for EOLC and LORC receivers. The cdf function can be interpreted as the outage probability for some threshold $\gamma$. The uncoded bit error rate can be easily found as [18]

$$
\mathrm{BER}_{x}=\frac{1}{2}\left[\sum_{i=1}^{P_{x}} A_{i}\left(1-\sqrt{\frac{\gamma_{x, i}}{1+\gamma_{x, i}}}\right)\right] .
$$

Similarly, if some of the eigenvalues in (69) are nondistinct, the pdf, cdf, and uncoded bit error rate expressions can easily be found by using partial fraction expansion.

\section{POWER CONSUMPTION SAVINGS ESTIMATE}

In this section we will try to give an estimate of the power saving due to the lower sampling rate and reduced processing load on the DSP achieved with the receiver that uses five-port device with respect to the power consumption of a conventional all-digital DS-CDMA receiver. The exact total amount of the power saving obtained with the proposed receiver could be made only after the receiver has been implemented. We will assume that it is not difficult to integrate a number of five-port devices in a single chip, so that their power consumption should not be significant.

As a "proof of the concept," we will use a DS-CDMA receiver with the following parameters: QPSK-modulated signal with the spreading factor $N=64$, oversampling ratio of $O=2$ samples per chip (for the conventional receiver), and a symbol rate of $R_{s}=1 \mathrm{Mbps}$.

\subsection{A/D conversion savings}

An all-digital DS-CDMA receiver requires two ADCs, one for $I$ and the other for $Q$ signal components, with a sampling rate of $f_{s 1}=R_{s} \times N \times O=128 \mathrm{Msps}$ each. On the other hand, a five-port-based RAKE receiver with three fingers requires at least 6 five-port devices for data detection and channel estimation (PN code tracking, if implemented by ana$\log$ circuitry, does not require A/D conversion even though it requires five-port circuits). Each five-port device is followed by at least two ADCs, which means that twelve ADCs with the sampling rate of $1 \mathrm{Msps}$ are required for the five-portdevice-based receiver. ${ }^{2}$ The approximate way of estimating the power consumption saving by using a lower sampling

\footnotetext{
${ }^{2}$ The number of symbol rate sampling ADCs can be smaller because many converters can sample more than one input signal. For example, 12 single-channel ADCs with the sampling rate of 1 Msps could be replaced with 3 four-channel ADCs with the sampling rate of 4 Msps.
} 
rate is to use the figure of merit for ADCs [20]:

$$
F=\frac{2^{\text {SNRbits }} f_{s}}{P_{\text {dis }}},
$$

where SNRbits represents the effective number of bits, $f_{s}$ is the sampling frequency, and $P_{\text {dis }}$ is the dissipation power. Assuming the same figure of merit for two different sampling frequencies, we form the ratio

$$
\frac{f_{s 1}}{f_{s 2}}=\frac{P_{\mathrm{dis} 1}}{P_{\mathrm{dis} 2}} .
$$

In our example, $f_{s 1} / f_{s 2}=P_{\text {dis } 1} / P_{\text {dis } 2}=128$, and after taking into account the number of $A / D$ converters, we have a power dissipation savings ratio of 21.3 in favour of analog despreading receiver. This number may be too optimistic, but significant power saving is to be expected nonetheless.

For the purpose of illustration, we will use an off-theshelf single-channel 10-bit AD9411 ADC by Analog Devices with the sampling rate of up to $170 \mathrm{Msps}$ [21], that could be used for the all-digital DS-CDMA receiver. Two such converters sampling at $128 \mathrm{Msps}$ consume about $2 \mathrm{~W}$. On the other hand, a 10-bit AD7440 single-channel converter with a total power dissipation of $9 \mathrm{~mW}$ at $1 \mathrm{Msps}$ can be used with each five-port device. twelve ADCs, required for a three-finger RAKE receiver, would consume about $0.108 \mathrm{~W}$ of power. This result is similar to the one obtained by using a figure of merit.

\subsection{DSP savings}

Once the signal is digitized with ADCs, the radio interface is implemented in software by means of digital signal processing, or in hardware by using ASIC or FPGAs. The most computationally intensive arithmetic operation that has to be done in the WCDMA receiver is the correlation with the spreading code. The complexity of correlation increases with the increase in spreading factor and with the number of RAKE fingers. We will use the same example to illustrate the reduction in the number of digital operations by moving the despreading in the analog domain. By following the similar methodology to the one outlined in [5], we find that the matched filter requires $2 \mathrm{NO}$ multiplications and $2 \mathrm{NO}$ additions per symbol. The output of the matched filter is downsampled to one sample per chip. Therefore, $4 \mathrm{~N}$ multiplications and $4 N$ additions are required to despread a complex signal. Thus, if we multiply this number by 4 to account for pilot, data, and two tracking correlators, we have $16 \mathrm{~N}+8 \mathrm{NO}$ floating point operations (FLOPS) per one data symbol per RAKE finger. The number of required FLOPS per symbol is 6.144 GFLOPS for a three-finger RAKE receiver. The estimate in [3], which is a result of a DARPA small unit operations project, shows that despreading/correlation accounts for $50 \%$ of the total number of operations at the DS-CDMA transceiver. Dynamic power consumption is proportional to the node switching activity [5], which means that about half of the power required for the DSP/ASIC can be saved by performing the analog instead of digital despreading.

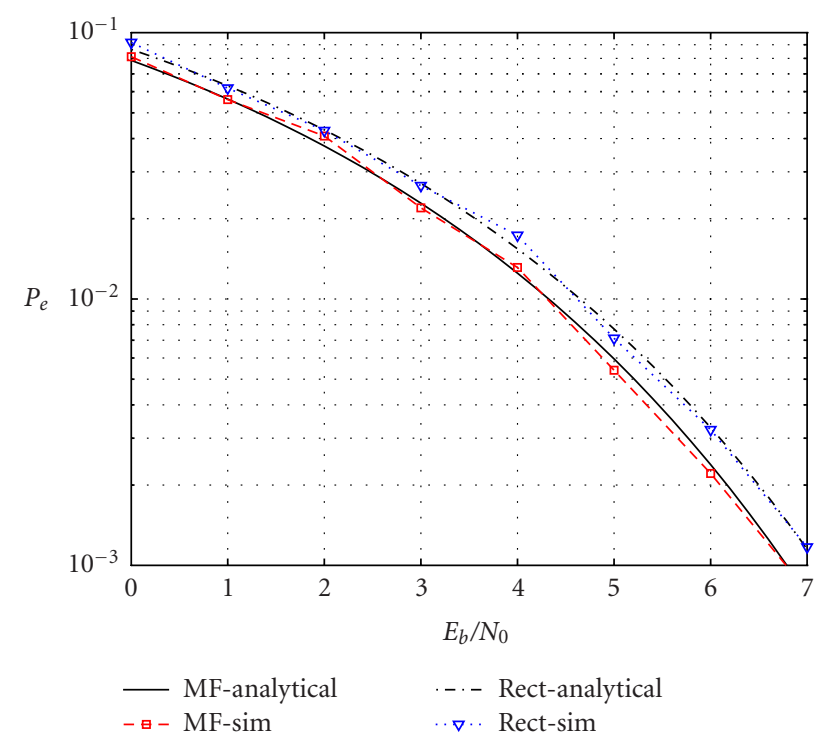

FIGURE 6: Uncoded BER with MF and rectangular despreading, $\alpha=$ $0.3, N=64$.

We have shown that there is potential power saving when a DS-CDMA receiver is designed by using a five-port device. On the other hand, five-port-based receiver would have some other losses. For example, replication of five-port devices and the need for additional PN code tracking circuitry would reclaim some portion of the power consumption savings discussed above. However, in addition to power consumption reduction, the five-port approach with analog despreading makes very high data rates possible that would otherwise be impossible due to technological limitations associated with sampling rates of ADCs and processing power of DSPs.

\section{NUMERICAL RESULTS}

The uncoded bit error rate of a single-finger receiver without fading when rectangular despreading is used is compared with the uncoded bit error rate of the receiver that uses chipmatched filter in Figure 6. Here we can see that the performance loss due to the rectangular despreading is minimal. Also, we see that the simulation results are in good agreement with the analytical expressions derived above. A 17-bit LFSR shift register is used for PN code generation in our simulation, and Hadamard code is used for orthogonalization. The chip oversampling ratio is 10 , and the number of chips per symbol is $N=64$.

The RMS jitter of the proposed PN code tracking loop is compared with the RMS jitter of the conventional coherent and noncoherent L2-norm-based loop in Figure 7. From here we can see that the performance of L1-DLL obtained through simulation is better than predicted analytically due to the overestimation of the noise power spectral density in the adopted analytical model, and slightly better than the performance of the L2-DLL. The effects of different values for $\phi$ have also been analyzed, and it has been observed that in spite of decrease of SNR for case when $\phi=0$, the filter 


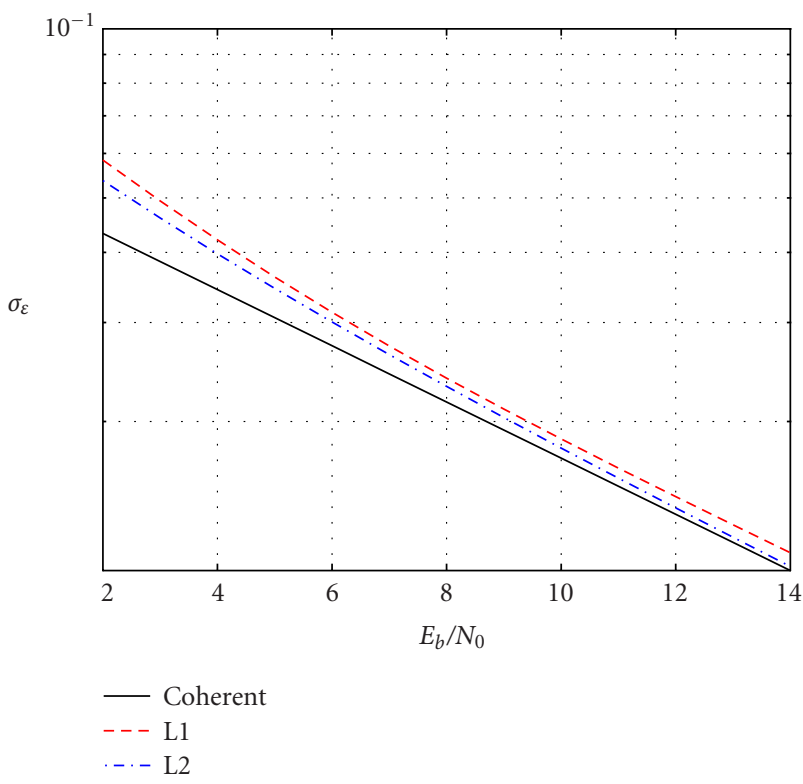

FIgURE 7: Normalized RMS jitter $\sigma_{\varepsilon_{X}}$, for $\Delta=T_{c} / 2$.

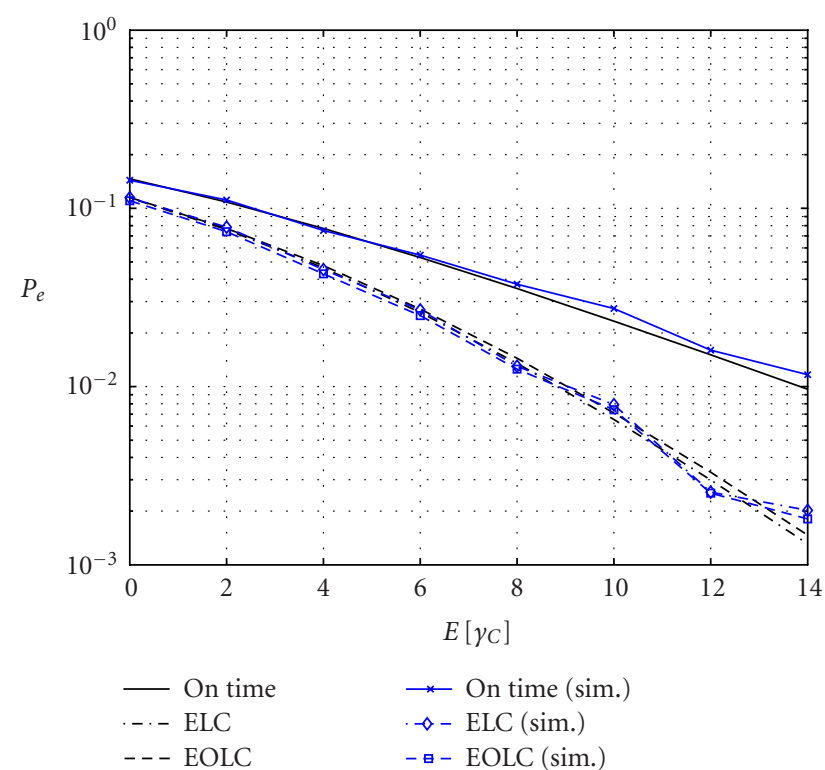

FIGURE 8: Bit error rates for conventional, ELC, and EOLC RAKE receivers under fast fading with $L=1, U_{1}=2, \tau_{2}-\tau_{1}=(1 / 2) T_{c}$, and $E\left[\left|a_{1}\right|^{2}\right] / E\left[\left|a_{2}\right|^{2}\right]=1, K_{u}=4 \mathrm{~dB}$.

see the comparison of uncoded bit error rates for three different receivers, namely, conventional, ELC, and EOLC combiners, under fast fading. Two unresolvable components are considered, spaced $1 / 2 T_{c}$ apart and their average power ratio is $S M R=1 \mathrm{~dB}$. The average SNR in the plot corresponds to $\overline{\gamma_{C}}$ for the best tau. Evidently, even the very simple receiver based on early and late correlators, or ELC combiner, outperforms the receiver based on the on-time correlation. This can be explained if we remember that there is a tracking bias due to the fast fading and presence of unresolvable multipath components. Thus, under fast fading scenario, it would be possible to use only two correlators that are also used for tracking, without a need for the on-time correlator. Also, we see a good agreement between the theoretical result and simulation. The self-noise and MAI do not have a significant impact on the receiver performance for a small number of users. For a given unresolvable multipath, the SNR gain achieved with the EOLC receiver, for the uncoded bit error rate of 0.01 , is $5 \mathrm{~dB}$. This performance gain is reduced to $1 \mathrm{~dB}$ for the case of slow fading, whereas the ELC receiver fares worse than the conventional receiver, as shown in Figure 9. The results discussed above are obtained for ideal, noiseless tracking and ideal channel estimation.

It is instructive to compare these receivers for different sampling instances, or tracking delay errors. Bit error rates of the three receivers with serially multiplexed pilot, for the fast fading case are shown in Figure 10. The average SNR at the on-time correlator is $\overline{\gamma_{C}}=10 \mathrm{~dB}$. Results for $\tau=0.2$ tracking error are in agreement with the results shown in Figure 8. Similarly, for the slow fading, Figure 11 shows that though the performance gain is not as significant as in the case of fast fading, the EOLC receiver is much less sensitive to the tracking error. 


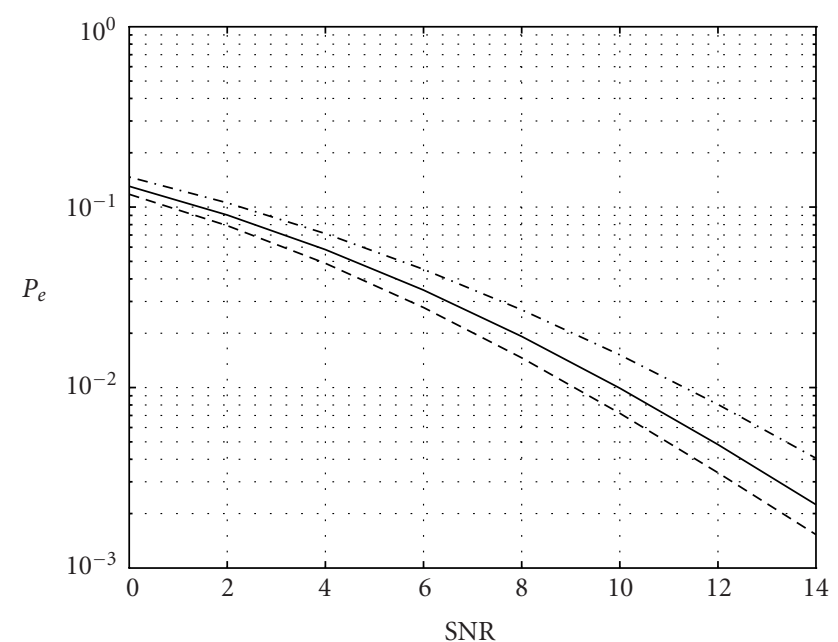

$$
\begin{aligned}
& \text { - On time } \\
& -\ldots \text { ELC } \\
& -- \text { EOLC }
\end{aligned}
$$

FIgURE 9: Bit error rates for conventional, ELC, and EOLC RAKE receivers under slow fading with $L=1, U_{1}=2, \tau_{2}-\tau_{1}=1 / 2 T_{c}$, and $E\left[\left|a_{1}\right|^{2}\right] / E\left[\left|a_{2}\right|^{2}\right]=1 \mathrm{~dB}$.

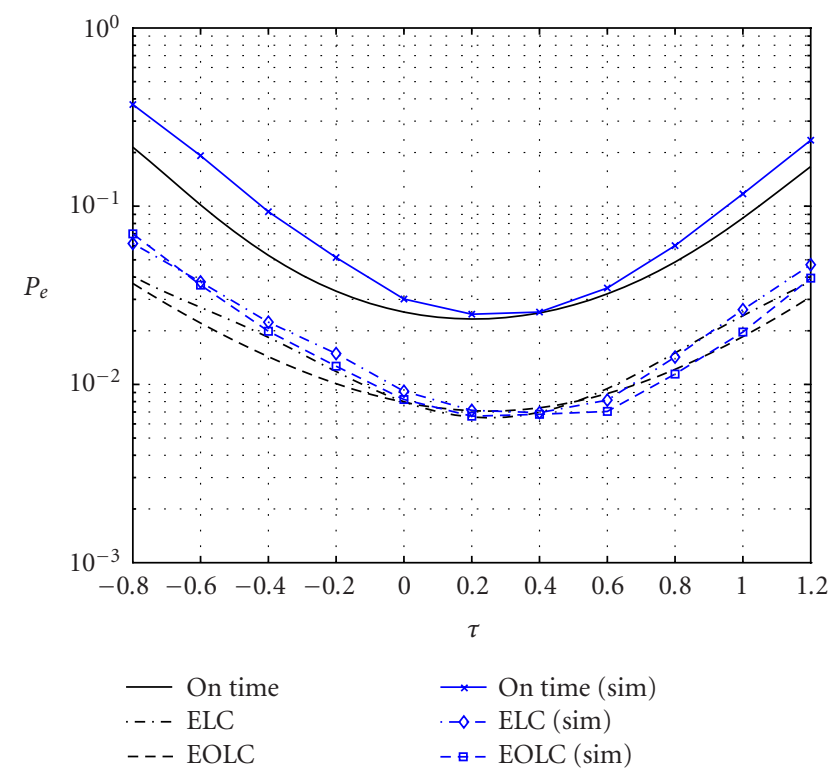

FIgURE 10: Comparison of analytical and simulation results for conventional, ELC, and EOLC RAKE receivers under fast Rayleigh fading with $L=1, U_{1}=2, \tau_{2}-\tau_{1}=0.5 T_{c}$, and $E\left[\left|a_{1}\right|^{2}\right] / E\left[\left|a_{2}\right|^{2}\right]=$ $1 \mathrm{~dB} ; \overline{\gamma_{C}}=10 \mathrm{~dB}, K_{u}=4$, and $N=64$.

The rest of the results is for the receiver with groups of fingers under the fast fading scenario. In Figure 12 we compare uncoded bit error rates versus SNR for the conventional and proposed LORC receiver, and in Figure 13 we compare BERs as functions of tracking errors. Obtained results are similar to the ones for the fractionally spaced fingers. Similarly, Figures 14 and 15 show results for the slow fading.

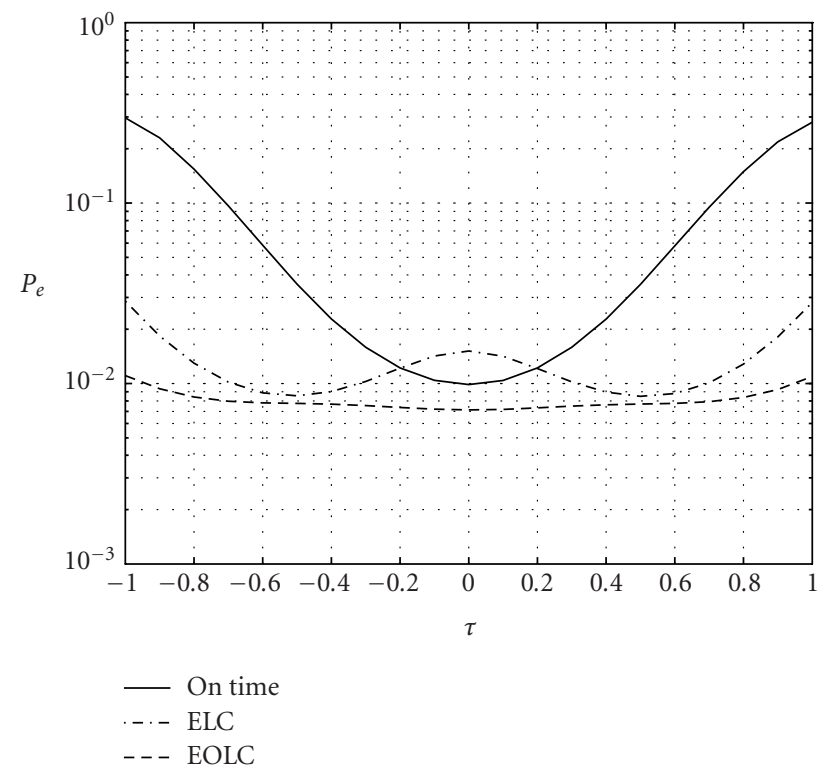

FIgURE 11: BER for conventional, ELC, and EOLC receivers under slow fading with $L=1, U_{1}=2, \tau_{2}-\tau_{1}=1 / 2 T_{c}$, and $E\left[\left|a_{1}\right|^{2}\right] / E\left[\left|a_{2}\right|^{2}\right]=1 \mathrm{~dB}$.

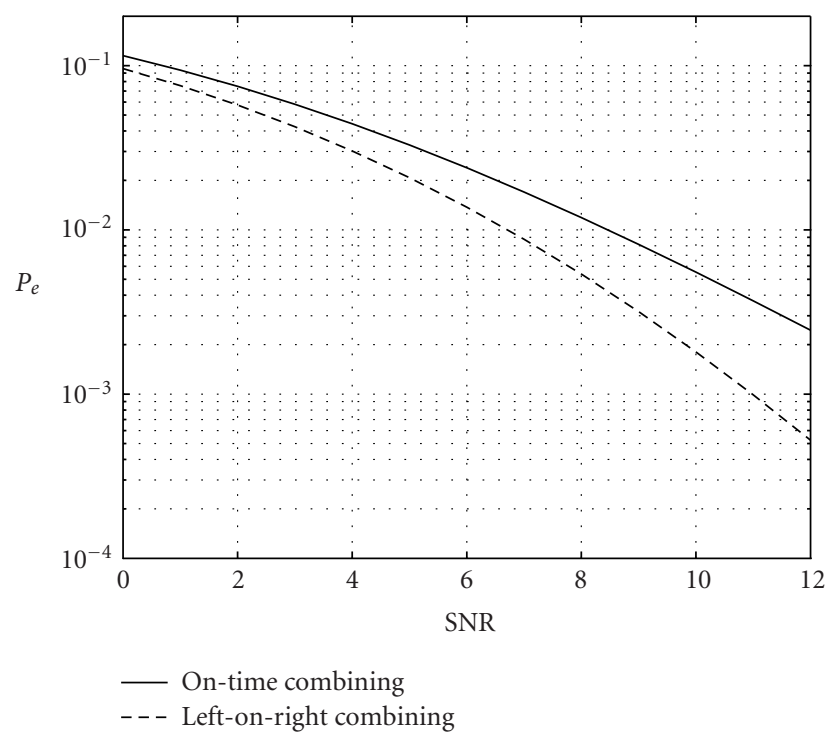

FIGURE 12: The BER comparison for conventional and LORC RAKE receivers under fast fading with $L=2$ resolvable multipath clusters, $U_{l}=2$ unresolvable components per cluster, $\tau_{i+1}-\tau_{i}=0.5 T_{c}$, and $E\left[\left|a_{i}\right|^{2}\right] / E\left[\left|a_{i+1}\right|^{2}\right]=3 \mathrm{~dB}$, for $i=0$ and $i=2$.

\section{CONCLUSIONS AND FURTHER WORK}

In this paper we propose a new low-complexity, low-power consumption DS-CDMA receiver that can be used for high data rate applications. The receiver is based on a passive five-port device. By proposing simultaneous direct conversion and analog despreading, we have potentially reduced the sampling rate by two orders of magnitude. The performance of the proposed receiver that uses rectangular instead 


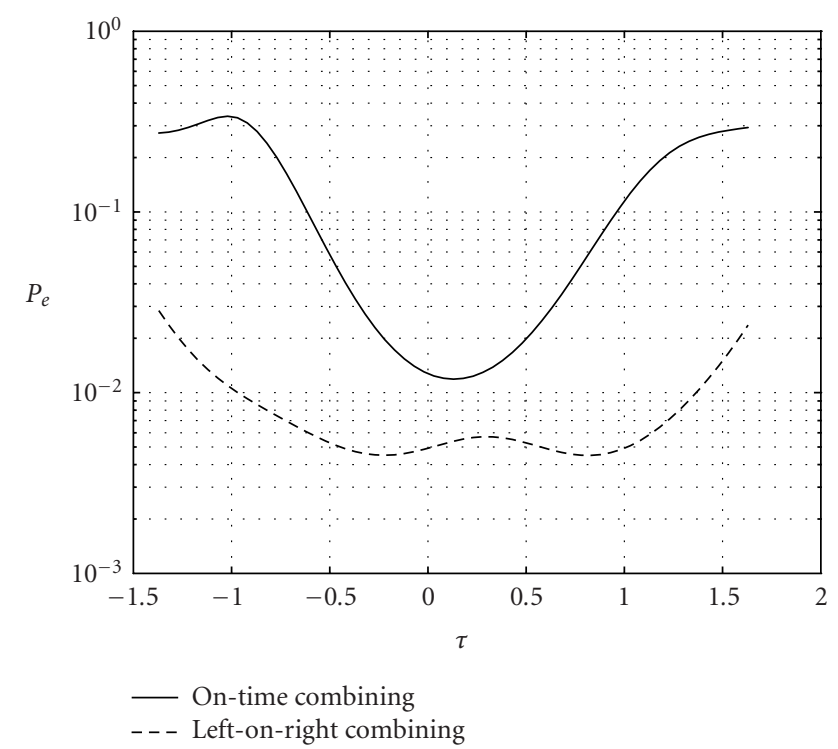

FIGURE 13: The BER comparison for conventional and proposed RAKE receivers under fast fading with $L=2$ resolvable clusters, $U_{l}=2$ unresolvable components per cluster, $\tau_{i+1}-\tau_{i}=0.5 T_{c}$, and $E\left[\left|a_{i}\right|^{2}\right] / E\left[\left|a_{i+1}\right|^{2}\right]=3 \mathrm{~dB}$, for $i=0$ and $i=2$; SNR $=8 \mathrm{~dB}$.

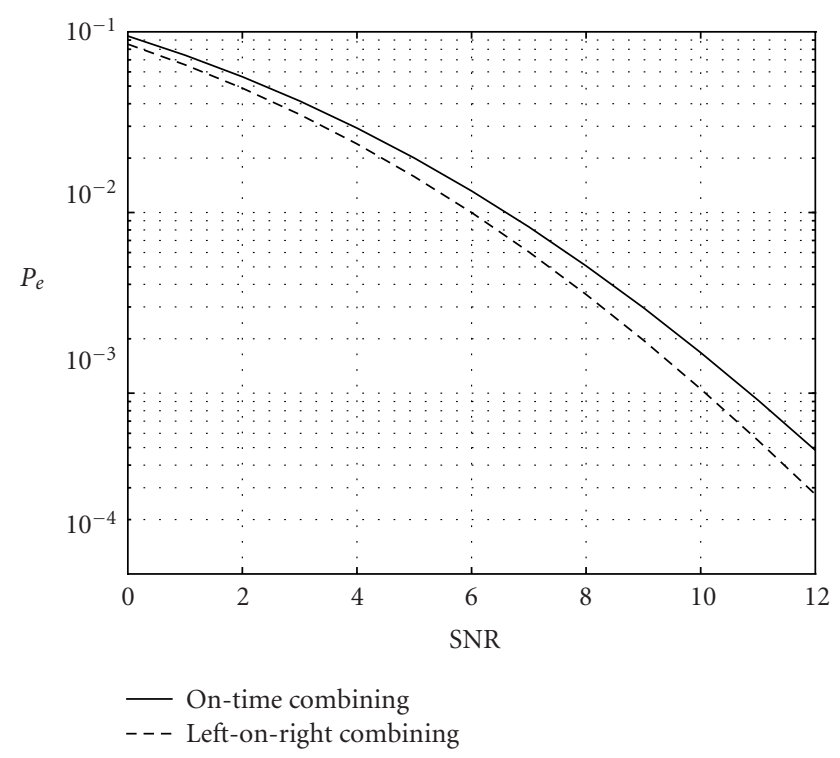

FIGURE 14: The BER comparison for conventional and proposed RAKE receivers under slow fading with $L=2$ resolvable multipath clusters, and $U_{l}=2$ unresolvable components per cluster, $\tau_{i+1}-\tau_{i}=0.5 T_{c}$, and $E\left[\left|a_{i}\right|^{2}\right] / E\left[\left|a_{i+1}\right|^{2}\right]=3 \mathrm{~dB}$, for $i=0$ and $i=2$.

of pulse-shaped despreading has been analyzed. Also, we propose a new noncoherent tracking scheme based on L1 norm that offers similar or even better performance than L2 norm tracking circuitry, at lower hardware complexity. Following that, we introduce two five-port-based RAKE receiver structures that result in better performance in terms of uncoded

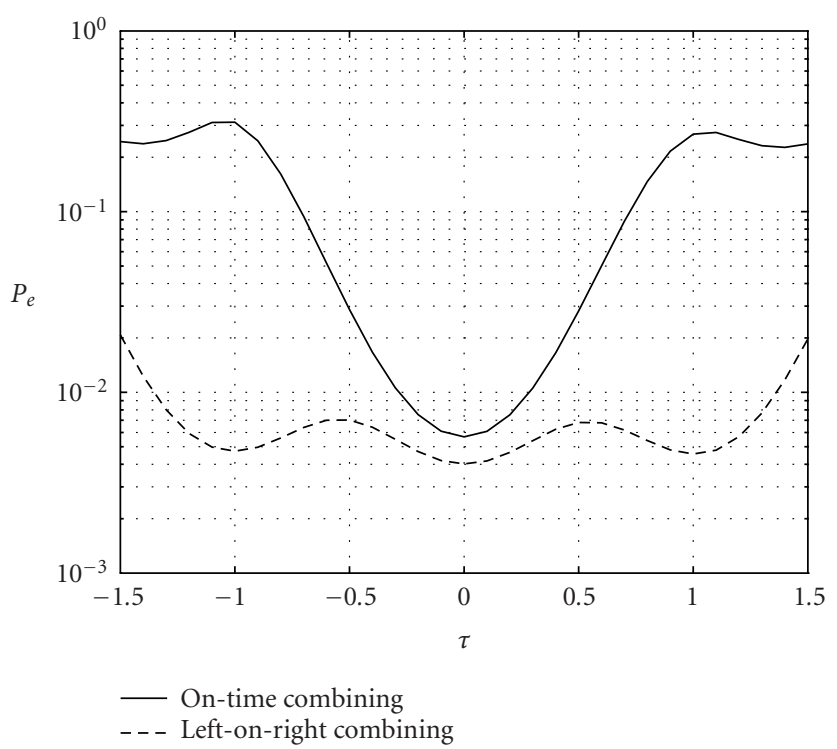

FIGURE 15: The BER comparison for conventional and proposed RAKE receivers under slow fading with $L=2$ resolvable clusters, $U_{l}=2$ unresolvable components per cluster, $\tau_{i+1}-\tau_{i}=0.5 T_{c}$, and $E\left[\left|a_{i}\right|^{2}\right] / E\left[\left|a_{i+1}\right|^{2}\right]=3 \mathrm{~dB}$, for $i=0$ and $i=2 ; \mathrm{SNR}=8 \mathrm{~dB}$.

bit error rate and tracking error sensitivity. Both fast and slow fading scenarios are considered. It has been shown that significant performance improvement with the proposed receiver structures is achieved in a fast fading scenario. The performance improvement for a slow fading is not as large. However, the advantage of the proposed receiver structures becomes evident when the tracking error is taken into account.

Many problems still remain open for further research. One of them is the problem of a limited dynamic range associated with the squaring operation performed in the five-port device. Possible solution may involve placing an analog adaptive gain control circuitry in front of five-port devices. This could be combined with some type of digital control that compensates for dc offset and other effects (e.g., caused by temperature variations) that may cause the nonlinear block to work outside the squaring region.

\section{APPENDICES}

\section{A. THERMAL NOISE}

We begin by writing the noise portion of the signal:

$$
\nu(n)=\frac{1}{\sqrt{N T_{c}}} \sum_{k=0}^{N-1} c_{n}^{*}(k) \int_{(n-1) T-T_{c} / 2+k T_{c}}^{(n-1) T-T_{c} / 2+(k+1) T_{c}} n_{l}(t) d t .
$$

It is easy to show that the noise variance can be expressed as a power spectral density integral:

$$
\sigma_{v}^{2}=T_{c} \int_{-\infty}^{\infty} \operatorname{sinc}^{2}\left(f T_{c}\right) S_{n}(f) d f
$$


where $S_{n}(f)$ is the power spectral density of the noise process, and $\operatorname{sinc}(x)=\sin (\pi x) /(\pi x)$. If the input noise process has an infinitely wide power spectral density of $S_{n}(f)=N_{0}$, the noise variance becomes

$$
\sigma_{v}^{2}=N_{0} T_{c} \int_{-\infty}^{\infty} \operatorname{sinc}^{2}\left(f T_{c}\right) d f=N_{0} .
$$

However, an infinite noise power spectral density is not a realistic scenario, because receivers normally have an image rejecting prefilter that limits the noise and interference within the band of interest. Therefore, after the prefiltering, the noise process is band-limited within the bandwidth of the signal of interest. The bandwidth of the desired wideband signal for square-root-raised cosine pulse waveforms, before despreading, is given by the expression

$$
B_{W}=\frac{1+\alpha}{2 T_{c}}
$$

where $\alpha$ is the excess bandwidth. If we can set the noise bandwidth to be $B_{N}=1 / T_{c}$, thus allowing for some margin, the noise power of the filtered version of the thermal noise is

$$
\sigma_{v}^{2}=N_{0} T_{c} \int_{-B_{N}}^{B_{N}} \operatorname{sinc}^{2}\left(f T_{c}\right) d f=0.903 N_{0}
$$

which means that the noise effect has been somewhat reduced by the rectangular spreading operation. This is only valid if the noise is band-limited.

\section{B. RAKE RECEIVER SNR}

The SNR in the expression (68) is given in a quadratic form which can easily be transformed into the quadratic form of uncorrelated complex Gaussian vectors [19]:

$$
\begin{aligned}
\gamma_{x} & =\frac{E_{b}}{I_{0}} \mathbf{s}_{x}{ }^{H} \mathbf{R}_{s_{x}}^{-1 / 2} \mathbf{R}_{s_{x}}^{1 / 2} \mathbf{R}_{w_{x}}^{-1} \mathbf{R}_{s_{x}}^{1 / 2} \mathbf{R}_{s_{x}}^{-1 / 2} \mathbf{s}_{x} \\
& =\frac{E_{b}}{I_{0}}\left(\mathbf{R}_{s_{x}}^{-1 / 2} \mathbf{s}_{x}\right)^{H} \mathbf{R}_{s_{x}}^{1 / 2} \mathbf{R}_{w_{x}}^{-1} \mathbf{R}_{s_{x}}^{1 / 2}\left(\mathbf{R}_{s_{x}}^{-1 / 2} \mathbf{s}_{x}\right) \\
& =\frac{E_{b}}{I_{0}}\left(\mathbf{U}_{x}{ }^{H} \mathbf{R}_{s_{x}}^{-1 / 2} \mathbf{s}_{x}\right)^{H} \boldsymbol{\Lambda}_{x}\left(\mathbf{U}_{x}{ }^{H} \mathbf{R}_{s_{x}}^{-1 / 2} \mathbf{s}_{x}\right) \\
& =\frac{E_{b}}{I_{0}} \sum_{i}\left|u_{x, i}\right|^{2} \lambda_{x, i}=\sum_{i}\left|u_{x, i}\right|^{2} \gamma_{x, i},
\end{aligned}
$$

where $\boldsymbol{\Lambda}_{x}$ and $\mathbf{U}_{x}$ are the diagonal and unitary decompositions of the matrix $\mathbf{R}_{s_{x}}^{1 / 2} \mathbf{R}_{w_{x}}^{-1} \mathbf{R}_{s_{x}}^{1 / 2}$, respectively, $u_{x, i}$ are the unit variance complex Gaussian random variables, and $\lambda_{x, i}$ are the elements of $\boldsymbol{\Lambda}_{x}$. Here we assumed ideal channel estimation. For receivers with nonfractional finger spacing, the noise correlation matrix $\mathbf{R}_{w_{x}}$ is an identity matrix, and so is its inverse $\mathbf{R}_{w_{x}}^{-1}$.

\section{ACKNOWLEDGMENT}

This work was funded in part by SOMA Networks, Inc.

\section{REFERENCES}

[1] J. Hagenauer, M. Moerz, and A. Schaefer, "Analog decoders and receivers for high speed applications," in Proc. International Zurich Seminar on Broadband Communications (IZS '02), pp. 3-1-3-8, Zurich, Switzerland, February 2002.

[2] S. Sheng and R. Brodersen, Low Power CMOS Wireless Communications: a Wideband CDMA System Design, Kluwer Academic, Boston, Mass, USA, 1998.

[3] J. E. Gunn, K. S. Barron, and W. Ruczczyk, "A low-power DSP core-based software radio architecture," IEEE J. Select. Areas Commun., vol. 17, no. 4, pp. 574-590, 1999.

[4] K. Onodera and P. R. Gray, "A 75 mW 128 MHz DS-CDMA baseband correlator for high-speed wireless applications," in Proc. Symposium on VLSI Circuits Digest of Technical Papers, pp. 117-118, June 1997.

[5] V. Chandrasekhar, F. Livingston, and J. Cavallaro, "Reducing dynamic power consumption in next generation DSCDMA mobile communication receivers," in Proc. IEEE 14th International Conference on Application-specific Systems, Architectures and Processors (ASAP '03), pp. 251-261, the Hague, the Netherlands, June 2003.

[6] M. Ratni, D. Krupezevic, Z. Wang, and J.-U. Jurgensen, "Broadband digital direct down conversion receiver suitable for software defined radio," in Proc. IEEE 13th International Symposium on Personal, Indoor, and Mobile Radio Communications (PIMRC '02), vol. 1, pp. 100-104, Lisbon, Portugal, September 2002.

[7] X. Xu, K. Wu, and R. G. Bosisio, "Software defined radio receiver based on six-port technology," in Proc. IEEE MTT-S International Microwave Symposium Digest, vol. 2, pp. 10591062, Philadelphia, Pa, USA, June 2003.

[8] X. Huang, M. Caron, and D. Hindson, "Adaptive I/Q regeneration in 5-port junction based direct receivers," in Proc. 5th Asia-Pacific Conference on Communications and 4th Optoelectronics and Communications Conference (APCC/OECC '99), vol. 1, pp. 717-720, Beijing, China, October 1999.

[9] H. Meyr, M. Moeneclaye, and S. A. Fechtel, Digital Communication Receivers: Synchronization, Channel Estimation, and Signal Processing, John Wiley \& Sons, New York, NY, USA, 1998.

[10] J. K. Holmes, Coherent Spread-Spectrum Systems, Wiley Interscience, New York, NY, USA, 1982.

[11] S. Thayaparan, T.-S. Ng, and J. Wang, "Half-sine and triangular despreading chip waveforms for coherent delay-locked tracking in DS/SS systems," IEEE Trans. Commun., vol. 48, no. 8 , pp. 1384-1391, 2000.

[12] B. W. Hart, R. D. J. van Nee, and R. Prasad, "Performance degradation due to code tracking errors in spread-spectrum code-division multiple-access systems," IEEE J. Select. Areas Commun., vol. 14, no. 8, pp. 1669-1679, 1996.

[13] R. De Gaudenzi, "Direct-sequence spread-spectrum chip tracking in the presence of unresolvable multipath components," IEEE Trans. Veh. Technol., vol. 48, no. 5, pp. 1573$1583,1999$.

[14] K. J. Kim, S. Y. Kwon, E. K. Hong, and K. C. Whang, "Effect of tap spacing on the performance of direct-sequence spreadspectrum RAKE receiver," IEEE Trans. on Commun., vol. 48, no. 6, pp. 1029-1936, 2000.

[15] I. Maljević and E. S. Sousa, "Performance degradation due to code tracking errors on a RAKE spread-spectrum receiver in the presence of unresolvable multipath," in Proc. IEEE 13th International Symposium on Personal, Indoor, and Mobile Radio Communications (PIMRC '02), vol. 4, pp. 1815-1819, September 2002. 
[16] M. Guenach and L. Vandendorpe, "Downlink performance analysis of a BPSK-based WCDMA using conventional rake receivers with channel estimation," IEEE J. Select. Areas Commun., vol. 19, no. 11, pp. 2165-2176, 2001.

[17] A. J. Viterbi, CDMA: Principles of Spread Spectrum Communication, Addison Wesley, Reading, Mass, USA, 1995.

[18] J. G. Proakis, Digital Communications, McGraw-Hill International Editions, New York, NY, USA, 3rd edition, 1995.

[19] A. M. Mathai and S. B. Provost, Quadratic Forms in Random Variables: Theory and Applications, Marcel Dekker, New York, NY, USA, 1992.

[20] R. H. Walden, "Analog-to-digital converter survey and analysis," IEEE J. Select. Areas Commun., vol. 17, no. 4, pp. 539-550, 1999.

[21] Analog device data sheets, http://www.analog.com.

Ivo Maljević was born in Bar, Serbia and Montenegro, on November 22, 1966. He received the B.S. degree from the University of Podgorica in 1991, the M.S. degree from the University of Belgrade in 1995, and the Ph.D. degree from the University of Toronto, Canada, in 2004, all in electrical engineering. From 1993 to 1997 he worked as a Research Engineer at Mihajlo Pupin Institute, Belgrade. In 1997 he joined Mo-

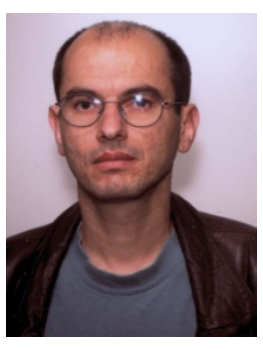
torola as a DSP software engineer, working on voice compression algorithms. Since 2004 he has been with Soma Networks, Toronto. His current research interests include CDMA systems, softwaredefined radio, signal processing, and digital communications theory.

Elvino S. Sousa holds the rank of Professor in the Department of Electrical and Computer Engineering, University of Toronto, Canada, and is the Bell University Lab Chair in computer engineering with a mandate in wireless communications. He is the Director of the Wireless Lab, which has undertaken research in spread spectrum systems and CDMA wireless systems for the past 16 years. His current research interests are in

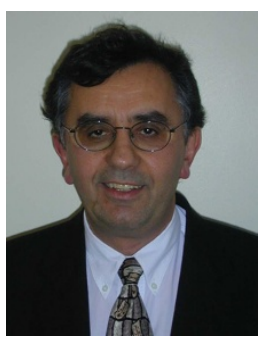
the areas of high-speed CDMA systems, smart antenna systems, software radio, ad hoc networks, and wireless system concepts for 4th-generation networks. Professor Sousa has been a Consultant to industry and governments in the area of wireless systems internationally. He was the Technical Program Chair for PIMRC '95, Vice-Technical Program Chair for Globecom '99, Chair of the IEEE Technical Committee on Personal Communications, and has been involved in the technical program committee of numerous international conferences. He has spent sabbatical leaves at Qualcomm and Sony CSL/ATL, where he was the holder of the Sony Sabbatical Chair. 